\title{
Squeezing of Optical States on the CCR-Algebra
}

\author{
By \\ Reinhard HONEGGER* and Alfred RIECKERS*
}

\begin{abstract}
Squeezing processes are commonly described in terms of quadratic Hamiltonians, which generate unitary implementations of Bogoliubov transformations of the quantized electromagnetic field. Here the behaviour of the quasifree, the classical, and the coherent photon states under general squeezing Bogoliubov transformations is investigated. It is found that there is a great variety of mixed classical states, which remain classical under the squeezing operation, whereas each pure classical state becomes non-classical. Especially, some classical. microscopic first order coherent states remain classical and coherent of first order under one-mode squeezing. This contrasts squeezing of macroscopic coherent states.
\end{abstract}

\section{\$1. Introduction}

Squeezed photon states constitute nowadays the main class of non-classical states of the quantized electromagnetic field. The experimental squeezing procedure starts usually with an easily preparable classical state, which oftenbut not necessarily - has some optical properties like a macroscopic phase and /or a certain degree of coherence. Thus it is an interesting theoretical question, under which kind of squeezing transformations such a state becomes non-classical.

The theoretical descriptions of squeezing processes are mostly derived from quadratic Hamiltonians of the photon field involving some classical, macroscopic pumping fields. The associated dynamics is given in terms of squeezing Bogoliubov transformations of the photon field observables [38], [24], [25], [26], [27].

The present investigation is devoted to the behaviour of some Boson state classes, which are frequently used in quantum optics, namely the quasifree, the classical, and the coherent states, under general squeezing Bogoliubov transformations. A systematic calculation of the associated variances of the

Communicated by H. Araki, November 10, 1995.

1991 Mathematics Subject Classifications: 81V80, 46N50, 47D45.

*Institut für Theoretische Physik, Universität Tübingen, D-72076 Tübingen, Germany. 
field expectation values is, however, performed in [26].

Our discussion is presented in terms of a rigorous smeared Boson field theory based on an arbitrary testfunction space $E$, a complex pre-Hilbert space. The choice of $E$ determines the specific Boson system and the number of modes taken into account. Both finitely and infinitely many field modes are covered in this way. For scalar Bosons $E$ is a subspace of $\mathbb{L}^{2}(\Lambda)$, whereas $E \subseteq \mathbb{L}^{2}(\Lambda) \mathbb{C}^{2 s+1}$ for Bosons with the spin $s,[3],[4]$. For photons the quantization procedure in the Coulomb gauge leads to a testfunction space $E$ consisting of divergence-free (i.e. $\nabla \cdot f=0$ ) functions $f: \Lambda \rightarrow \mathbb{C}^{3}$ on the quantization volume $\Lambda \subseteq \mathbb{R}^{3}$ in position space, equipped with the scalar product $\langle f \mid g\rangle=\int_{\Lambda} \overline{f(x)} \cdot g(x) \mathrm{d}^{3} x$ for $f, g \in E$, where $a \circ b=a_{1} b_{1}+a_{2} b_{2}+a_{3} b_{3}$ for $a=\left(a_{1}, a_{2}, a_{3}\right), b=\left(b_{1}, b_{2}, b_{3}\right) \in \mathbb{C}^{3}$, [7], [15], [10]. The smearing of the Boson field operators $\Phi(f)$ is not only indispensable for their mathematical realization but also clarifies their role as observables, where the testfunction $f \in E$ determines in which spatial region and in which (test-) mode a state of the quantized field is to be measured.

Squeezing Bogoliubov transformations of the Boson field arise from symplectic transformations $T$ on the one-Boson testfunction space $E$ with non-zero anti-linear part, which implies that at least one testmode is squeezed, e.g., in the vacuum state (cf. Subsection 2.3). For the present purposes the symplectic transformations are analyzed in Subsection 2.2 .

For the description of the (non-squeezed and squeezed) Boson states we use the techniques of generating functions (cf. Subsection 2.1), that are quantities, which are independent of any Hilbert space representation (and circumvent the problem of inequivalent representations of the field algebra) and provide a direct connection to the usual (non-smeared) field formalism [25].

As mentioned above classical states are those states which are most easily prepared in experiments. For example, the optical coherent photon states of a Maser or a Laser are classical, and so are the thermal equilibrium states, which in addition are quasifree (see the Sections 3, 4, and 5; cf. also the pioneering work [3]). In our approach the classical states are characterized by the positive definiteness of the normally ordered generating functions, and they turn out to be the mixtures of the generalized pure, coherent states (see Proposition $3.3)$. They correspond to states with a positive $P$-representation and obey the classical correlation inequalities [9], [37]. For applications it is central to describe precisely how the squeezing operations act on classical states.

We show in Section 3 that under a squeezing Bogoliubov transformation all pure classical states become non-classical. But there exists a great variety of (mixed) classical states which remain classical under the squeezing operation. This is an effect, which is demonstrated in terms of quasifree classical states in Section 4, and which up to now does not seem to have been analyzed in the literature. 
In Section 5 the squeezing theory of classical coherent states is worked out, which is based on our previous generalization and refinement of Glauber's coherence theory [9] in terms of smeared fields [21], [22], [19]. In a coherent many photon state the expectation of the Boson field operator $\Phi(f)$ with testfunction $f \in E$ is given by means of $\operatorname{Re}(L(f))$, where $L-$ a complex-linear form on the testfunction space $E$ - plays the role of Glauber's complex coherence function (after smearing). In this connection $\operatorname{Re}(L(f))$ (with testfunction $f \in E$ varied) describes the shape of the effective classical field produced by the coherent state. This interpretation of the (smeared) coherence function $L$, following from the basic notions of the theory (which is, however, not made explicit in the quantum optical text books [10], [31], [29], [32]), is used here to discuss the physical aspects of the squeezing procedure. There are to be discriminated the two cases, where the norm $\|L\|$ of $L$ is finite $(L$ is then a bounded linear form) or not (unbounded linear form). In the first case, called microscopic coherence, the coherent state is representable by a density operator in the Fock space, whereas in the second case, named macroscopic coherence, non-Fock representations have to be used. The naming derives mainly from the observation, that the particle number expectation value in a coherent state is proportional to $\|L\|^{2}$, a relation which reconciles the photon (particle) aspects with those of a classical field. In position space a finite $\|L\|$ expresses mathematically square integrability and physically it means localization and boundedness of the radiation peaks. For macroscopic radiation states, however, a sufficient intensity is expected on surfaces far from the radiation center, preventing a finite $\|L\|$.

As initial states to be squeezed we take in Section 5 the classical first order coherent states $\mathscr{\ell}_{L}^{(1)}$ associated with the coherence function $L$. We show that under a squeezing Bogoliubov transformation every macroscopic coherent state (with $\|L\|=\infty$ ) is rendered non-classical and no longer is coherent. For microscopic coherent states (with $\|L\|<\infty$ ) there are, however, completely natural looking cases, which remain classical and coherent-with a transformed coherence function- under a one-mode squeezing operation.

The latter case is further analyzed in Section 6, where for a given bounded coherence function $L$ a one-parameter family of squeezing transformations is constructed, which only squeezes (and rotates) the mode $L$, whereas all other testmodes are only rotated without squeezing. There is then a critical squeezing strength, above which all states in $\mathscr{S}_{L}^{(1)}$ are rendered non-classical and below which some states from $\mathscr{S}_{L}^{(1)}$ remain classical. For the latter situation a one-parameter family of examples, consisting of Gaussian (i.e., quasifree) classical coherent states is constructed. However, independently of the squeezing strength every transformed state possesses still quantum optical coherence of first order. 


\section{§2. Basic Notions and Preliminary Results}

Throughtout the present paper we assume $\mathscr{H}$ to be an arbitrary complex Hilbert space with (right-linear) scalar product $\langle\cdot \mid \cdot\rangle$, and $E$ to be a dense complex subspace of $\mathscr{H}$. With $A \subseteq B$ it is denoted the set inclusion, for a proper inclusion we write $A \subset B$. The orthogonal complement of a subset $K \subseteq \mathscr{H}$ with respect to $\langle\cdot \mid \cdot\rangle$ is denoted by $K^{\perp}$, and $\operatorname{dim}(K)$ is the complex dimension of the complex subspace $K \subseteq \mathscr{H}$.

\subsection{States and Representations}

For the convenience of the reader let us here repeat the basic notions of the $\mathrm{C}^{*}$-Boson field theory. The complex pre-Hilbert space $E$ is considered as the one-Boson testfunction space taking into account the test modes of the Boson field. The $\mathrm{C}^{*}$-algebra of the Boson system is taken as the Weyl algebra $\mathbb{W}(E)$ over $E$. $W(E)$ is generated by nonzero elements $W(f), f \in E$, - the Weyl operators - satisfying the Weyl relations (cf. [4, Theorem 5.2.8])

$$
W(f) W(g)=\exp \left\{-\frac{i}{2} \operatorname{Im}\langle f \mid g\rangle\right\} W(f+g), W(f)^{*}=W(-f) \quad \forall f, g \in E .
$$

$\mathscr{\&} \equiv \mathscr{W}(E))$ denotes the state space of $\mathscr{W}(E)$, which is convex and weak $^{*}$-compact. The elements of the extreme boundary $\partial_{e} \&$ of $\&$ are the pure states. Each $\omega \in \mathscr{S}$ is uniquely determined by its characteristic function [20]

$$
C_{\omega}: E \rightarrow \mathbb{C}, f \longrightarrow C_{\omega}(f):=\langle\omega ; W(f)\rangle,
$$

the expectation values of the Weyl operators. We denote by $\mathscr{C}(E)$ the convex set of functions $C: E \rightarrow \mathbb{C}$ with $C(0)=1$ and for which the map $(f, g) \mapsto$ $\exp \left\{\frac{i}{2} \operatorname{Im}\langle f \mid g\rangle\right\} C(g-f)$ constitutes a positive definite kernel $E \times E \rightarrow \mathbb{C}([8]$, cf. also the Appendix of [20]). From [20] we cite the following result.

Lemma 2.1. The map $C: \& \rightarrow \mathscr{C}(E), \omega \mapsto C_{\omega}$, is affine and bijective. Moreover, we have the continuity relation: $\lim _{i} \omega_{\imath}=\omega$ in the weak ${ }^{*}-$ topology, if and only if $\lim _{\imath} C_{\omega_{\imath}}(f)=C_{\omega}(f) \forall f \in E$.

The characteristic function $C_{\mathrm{vac}}$ of the (Fock) vacuum state $\omega_{\mathrm{vac}} \in \&$ is given by

$$
C_{\mathrm{vac}}(f):=\left\langle\omega_{\mathrm{vac}} ; W(f)\right\rangle=\exp \left\{-\frac{1}{4}\|f\|^{2}\right\} \quad \forall f \in E .
$$

A state $\omega \in \mathscr{S}$ is called regular, if for each $f \in E$ the map $t \in \mathbb{R} \rightarrow C_{\omega}(t f)$ is continuous, and $\omega$ is said to be of class $\mathscr{C}^{m}$, if $t \in \mathbb{R} \rightarrow C_{\omega}(t f)$ is $m$-times differentiable for every $f \in E$, where $m \in \mathbb{N} \cup\{\infty\} . \omega$ is called analytic, if for each $f \in E$ the function $t \rightarrow C_{\omega}(t f)$ is analytic in a neighbourhood of the origin. If for every $f \in E$ this neighbourhood is all of $\mathbb{R}$, then $\omega$ is called entive-analytic. 
Especially, since $z \in \mathbf{C} \rightarrow \exp \left\{-\frac{z^{2}}{4}\|f\|^{2}\right\}$ is entire-analytic, so is the vacuum state $\omega_{\text {vac. }}$

In the GNS-representation $\left(\Pi_{\omega}, \mathscr{H}_{\omega}, \Omega_{\omega}\right)$ of the regular state $\omega$ on $\mathscr{W}(E)$ the existence of selfadjoint field operators $\Phi_{\omega}(f)$, that is, $\Pi_{\omega}(W(t f))=$ $\exp \left\{i t \Phi_{\omega}(f)\right\} \forall t \in \mathbf{R}$, is ensured by Stone's theorem. They fulfill the canonical commutation relations (CCR)

$$
\left[\Phi_{\omega}(f), \Phi_{\omega}(g)\right] \subseteq i \operatorname{Im}\langle f \mid g\rangle \mathbb{1} \forall f, g \in E
$$

on suitable dense domains. $f \in E \mapsto \Phi_{\omega}(f)$ is real-linear. The smeared annihilation and creation operators, $a_{\omega}(f):=\sqrt{2}^{-1}\left(\Phi_{\omega}(f)+i \Phi_{\omega}(i f)\right)$ and $a_{\omega}^{*}(f):=$ $\sqrt{2}^{-1}\left(\Phi_{\omega}(f)-i \Phi_{\omega}(i f)\right)$, associated with $\omega$ are densely defined and closed. It holds $a_{\omega}(f)^{*}=a_{\omega}^{*}(f)$, the mapping $f \mapsto a_{\omega}(f)$ is anti-linear and $f \mapsto a_{\omega}^{*}(f)$ is linear. The commutation relations

$$
\left[a_{\omega}(f), a_{\omega}(g)\right]=\left[a_{\omega}^{*}(f), a_{\omega}^{*}(g)\right]=0,\left[a_{\omega}(f), a_{\omega}^{*}(g)\right] \subseteq\langle f \mid g\rangle \mathbb{1}
$$

follow from $(2.2)$, cf. [4].

If $\omega \in \mathscr{S}$ is of class $\mathscr{C}^{2 m}$, then the associated cyclic vector $\Omega_{\omega}$ is contained in the domain of each polynomial of field operators with degree $\leq m$, in which case one defines

$$
\left\langle\omega ; \Phi_{\omega}\left(f_{1}\right) \cdots \Phi_{\omega}\left(f_{2 m}\right)\right\rangle:=\left\langle\Phi_{\omega}\left(f_{m}\right) \cdots \Phi_{\omega}\left(f_{1}\right) \Omega_{\omega} \mid \Phi_{\omega}\left(f_{m+1}\right) \cdots \Phi_{\omega}\left(f_{2 m}\right) \Omega_{\omega}\right\rangle .
$$

If $\omega \in \mathscr{S}$ is of class $\mathscr{C}^{2}$, then for each field operator $\Phi_{\omega}(f), f \in E$, one may calculate the variance (fluctuation) of the expectation value

$$
\begin{aligned}
\operatorname{Var}(\omega ; f): & =\left\langle\omega ;\left(\Phi_{\omega}(f)-\left\langle\omega ; \Phi_{\omega}(f)\right\rangle\right)^{2}\right\rangle=\left\langle\omega ; \Phi_{\omega}(f)^{2}\right\rangle-\left\langle\omega ; \Phi_{\omega}(f)\right\rangle^{2} \\
& =\left(\left.\frac{\mathrm{d} C_{\omega}(t f)}{\mathrm{d} t}\right|_{t=0}\right)^{2}-\left.\frac{\mathrm{d}^{2} C_{\omega}(t f)}{\mathrm{d} t^{2}}\right|_{t=0}
\end{aligned}
$$

(cf. [16]) with a finite result.

Analytic states are characterized by the following result, which is due to $[20]$.

Lemma 2.2. Let $\omega \in \mathscr{S}$. Then $\omega$ is analytic, if and only if for each $f \in E$ there exists a neighbourhood $U_{f} \subseteq \mathbb{C}$ of the origin (with $U_{f}=\overline{U_{f}}$ ) and an analytic function $U_{f} \times U_{f} \ni(u, v) \mapsto N_{\omega}(u, v ; f)$, such that

$$
C_{\omega}(u f)=C_{\mathrm{vac}}(u f) N_{\omega}(u, \bar{u} ; f) \quad \forall u \in U_{f} .
$$

Especially $\omega$ is entire analytic, if and only if $U_{f}$ may be chosen as the whole of $\mathbf{C}$ for every $f \in E$.

Furthermore, the analyticity condition for $\omega$ and (2.3) determine $N_{\omega}(u, v ; f)$ to be uniquely given by its Taylor series 


$$
N_{\omega}(u, v ; f)=\sum_{k, l=0}^{\infty}\left(\frac{i u}{\sqrt{2}}\right)^{k}\left(\frac{i v}{\sqrt{2}}\right)^{l} \frac{1}{k !} \frac{1}{l !}\left\langle\omega ; a_{\omega}^{*}(f)^{k} a_{\omega}(f)^{l}\right\rangle, \quad u, v \in U_{f},
$$

from which one obtains the normally ordered expectations according to

$$
\left\langle\omega ; a_{\omega}^{*}(f)^{k} a_{\omega}(f)^{l}\right\rangle=\left.\left(\frac{\sqrt{2}}{i}\right)^{k+l} \frac{\partial^{k+l} N_{\omega}(u, v ; f)}{\partial u^{k} \partial^{l}}\right|_{u=v=0^{\prime}}, \quad \forall k, l \in \mathbb{N}_{0} .
$$

For the (possibly non-analytic) state $\omega \in \&$ the above Lemma suggests the notion of a normally ordered characteristic function for the mapping

$$
P_{\omega}: E \rightarrow \mathbb{C}, f \mapsto P_{\omega}(f):=\exp \left\{\frac{1}{4}\|f\|^{2}\right\} C_{\omega}(f)=\frac{C_{\omega}(f)}{C_{\mathrm{vac}}(f)} .
$$

This notion is in accordance with the quantum optics literature [7], [32], cf. also the Appendix A.2 of [26].

\subsection{Symplectic Treamsiformations}

The imaginary part of the scalar product is a non-degenerate symplectic form on $E$.

Definition 2.3 (Symplectic Transfiormation) 。A mapping $T: E \rightarrow E$ is called a symplectic transformation on $E$, if it is real-linear, if $T(E)=E$. and if it fulfills

$$
\operatorname{Im}\langle T f \mid T g\rangle=\operatorname{Im}\langle f \mid g\rangle \quad \forall f, g \in E .
$$

We denote the set of all symplectic transformations on $E$ by $\mathscr{T}(E)$.

Obviously (2.6) implies the injectivity of $T$, and thus each symplectic transformation $T$ acts bijectively on $E$. Thus $T^{-1} \in \mathscr{T}(E)$, and $\mathscr{T}(E)$ forms a group.

Since $E$ and $\mathscr{H}$ are complex-linear vector spaces we may decompose each real-linear operator $T: E \rightarrow \mathscr{H}$ into its (complex-) linear part $T_{l}$ and its (complex-) anti-linear part $T_{a}$, that is, $T=T_{l}+T_{a}$ with the unique expressions

$$
T_{l}:=\frac{1}{2}(T-i T i) \quad \text { and } \quad T_{a}:=\frac{1}{2}(T+i T i) .
$$

In the following we consider $T_{l}$ and $T_{\boldsymbol{a}}$ for a symplectic $T \in \mathscr{T}(E)$ as (not necessarily bounded) operators on the Hilbert space $\mathscr{H}$ with the dense domain of definition $E$ and range contained in $E$. We mention that the adjoint $B^{*}$ of the densely defined operator $B$ on $\mathscr{H}$ is given by $\left\langle B^{*} g \mid f\right\rangle=\langle g \mid B f\rangle$ for linear $B$, and by $\left\langle f \mid B^{*} g\right\rangle=\langle g \mid B f\rangle$ for anti-linear $B, f \in \mathscr{D}(B)$ and $g \in \mathscr{D}\left(B^{*}\right)$. The restriction of the operator $B$ to the subspace $K \subseteq \mathscr{D}(B)$ is denoted by $\left.B\right|_{K}$, and $\operatorname{ker}(B)$ is the kernel of $B$.

A complete characterization of a symplectic $T \in \mathscr{T}(E)$ is found in [24]. For 
the present purposes, however, we need the following lemma.

Lemma 2.4. Let $T \in \mathscr{T}(E)$. Then it holds

$$
T_{l}^{*} \supseteq \frac{1}{2}\left(T^{-1}-i T^{-1} i\right), T_{a}^{*} \supseteq-\frac{1}{2}\left(T^{-1}+i T^{-1} i\right),
$$

implying $T^{-1}=\left.T_{l}^{*}\right|_{E}-\left.T_{a}^{*}\right|_{E}$ with $\left(T^{-1}\right)_{l}=\left.T_{l}^{*}\right|_{E}$ and $\left(T^{-1}\right)_{a}=-\left.T_{a}^{*}\right|_{E}$. Further,

$$
\begin{gathered}
T_{l}^{*} T_{a}=T_{a}^{*} T_{l}, \quad T_{l}^{*} T_{l}-T_{a}^{*} T_{a}=\mathbb{1}_{E}, \\
\left.T_{l} T_{a}^{*}\right|_{E}=\left.T_{a} T_{l}^{*}\right|_{E},\left.T_{l} T_{l}^{*}\right|_{E}-\left.T_{a} T_{a}^{*}\right|_{E}=\mathbb{1}_{E} . \\
T\left(\operatorname{ker}\left(T_{a}\right)\right)=\operatorname{ker}\left(\left.T_{a}^{*}\right|_{E}\right)
\end{gathered}
$$

Moreover, for each complex subspace $F$ of $E$ it follows:

(a) The subsequent three properties are equivalent:

(i) $F \subseteq \operatorname{ker}\left(T_{a}\right)$,

(ii) $\|T f\|=\|f\| \forall f \in F$,

(iii) $T$ acts (complex-) linearly on $F$.

Especially: $T_{a}=0 \Leftrightarrow T$ is unitary $\Leftrightarrow T$ is linear on $E$.

(b) The subsequent two assertions are equivalent:

(i) $\left.T_{a}^{*}\right|_{F} \neq 0$,

(ii) there is an $g_{0} \in F$ with $\left\|g_{0}\right\|<\left\|T^{-1} g_{0}\right\|$.

(c) The subsequent two assertions are equivalent:

(i) $\left.T_{a}\right|_{F} \neq 0$,

(ii) there is an $f_{0} \in F$ with $\left\|f_{0}\right\|<\left\|T f_{0}\right\|$.

Proof. From (2.6) it follows that $\operatorname{Im}\langle f \mid T g\rangle=\operatorname{Im}\left\langle T^{-1} f \mid g\right\rangle \forall f, g \in E$. With the use of $\langle f \mid g\rangle=\operatorname{Im}\langle-i f \mid T g\rangle+i \operatorname{Im}\langle f \mid g\rangle \forall f, g \in E$ one calculates

$$
\langle f \mid(T-i T i) g\rangle=\left\langle\left(T^{-1}-i T^{-1} i\right) f \mid g\right\rangle, \quad\langle f \mid(T+i T i) g\rangle=-\left\langle g \mid\left(T^{-1}+i T^{-1} i\right) f\right\rangle,
$$

which leads to (2.8). Inserting $T=T_{l}+T_{\boldsymbol{a}}$ in (2.6) and taking the adjoints leads to

$$
\operatorname{Im}\left\langle T_{l}^{*} T_{l} f \mid g\right\rangle+\operatorname{Im}\left\langle T_{l}^{*} T_{a} f \mid g\right\rangle+\operatorname{Im}\left\langle g \mid T_{a}^{*} T_{a} f\right\rangle+\operatorname{Im}\left\langle g \mid T_{a}^{*} T_{l} f\right\rangle=\operatorname{Im}\langle f \mid g\rangle \forall f, g \in E .
$$

The relation $\operatorname{Im}\langle f \mid g\rangle=-\operatorname{Im}\langle g \mid f\rangle$ and the non-degeneracy of $\operatorname{Im}\langle\cdot \mid \cdot\rangle$ finally gives

$$
(\underbrace{T_{l}^{*} T_{l}-T_{a}^{*} T_{a}-1_{E}}_{\text {linear }}) f+(\underbrace{T_{l}^{*} T_{a}-T_{a}^{*} T_{l}}_{\text {anti-linear }}) f=0 \forall f \in E .
$$

Since $E$ is a complex-linear vector space, we may replace $f$ by if, which gives (2.9). (2.10) is obtained by replacing $T$ by $T^{-\mathbf{1}}$.

(a): (2.9) implies 


$$
\|T f\|^{2}-\|f\|^{2}=2\left\|T_{a} f\right\|^{2}+2 \operatorname{Re}\left\langle T_{l} f \mid T_{a} f\right\rangle \forall f \in E,
$$

which proves $(\mathrm{i}) \Rightarrow$ (ii). (ii) $\Rightarrow$ (iii): For each $f \in F$ there is a $\theta \in \mathbb{R}$ such that

$$
\left|\left\langle T_{l} f \mid T_{a} f\right\rangle\right|=\operatorname{Re}\left(\mathrm{e}^{-i 2 \theta}\left\langle T_{l} f \mid T_{a} f\right\rangle\right)=\operatorname{Re}\left\langle T_{l} \mathrm{e}^{i \theta} f \mid T_{a} \mathrm{e}^{i \theta} f\right\rangle .
$$

But $\mathrm{e}^{i \theta} f \in F$, since $F$ is a complex vector space. Thus the assumption $\left\|T \mathrm{e}^{i \theta} f\right\|=$ $\left\|e^{i \theta} f\right\|$ implies with (2.12) that $\left\|T_{a} f\right\|^{2}+\left|\left\langle T_{l} f \mid T_{a} f\right\rangle\right|=0 \forall f \in F$. Hence $\left.T_{a}\right|_{F}=0$, and $\left.T\right|_{F}=\left.T_{l}\right|_{F}$ is linear. If (iii) is valid, then $i T$ if $=-T f \forall f \in F$ and thus $T_{a} f=0$ by $(2.7)$, which coincides with (i).

(b) : (i) $\Rightarrow$ (ii): (2.12) is also valid for $T^{-1}=\left.T_{l}^{*}\right|_{E}-\left.T_{a}^{*}\right|_{E}$. For all $f \in F$, assume $\|f\| \geq\left\|T^{-1} f\right\|$, which yields $\left\|T_{a}^{*} f\right\|^{2}-\operatorname{Re}\left\langle T_{l}^{*} f \mid T_{a}^{*} f\right\rangle \leq 0 \forall f \in F$. But for each $f$ $\in F$ there is a $\phi \in \mathbb{R}$ such that

$$
\left|\left\langle T_{l}^{*} f \mid T_{a}^{*} f\right\rangle\right|=-\operatorname{Re}\left(\mathrm{e}^{-i 2 \phi}\left\langle T_{l}^{*} f \mid T_{a}^{*} f\right\rangle\right)=-\operatorname{Re}\left\langle T_{l}^{*} \mathrm{e}^{i \phi} f \mid T_{a}^{*} \mathrm{e}^{i \phi} f\right\rangle .
$$

Thus $\left\|T_{a}^{*} f\right\|^{2}+\left|\left\langle T_{l}^{*} f \mid T_{a}^{*} f\right\rangle\right| \leq 0 \quad \forall f \in F$, which leads to $T_{a}^{*} f=0 \quad \forall f \in F$, a contradiction. (ii) $\Rightarrow$ (i) follows from (a) applied to $T^{-1}$.

(2.11): $T$ acts linearly on $\operatorname{ker}\left(T_{a}\right)$. Thus $T^{-1}$ acts linearly on $T\left(\operatorname{ker}\left(T_{a}\right)\right)$. Using (a) (iii) $\Rightarrow$ (i) for $T^{-1}$ with $F:=T\left(\operatorname{ker}\left(T_{a}\right)\right)$ implies that $T\left(\operatorname{ker}\left(T_{a}\right)\right) \subseteq$ $\operatorname{ker}\left(\left.T_{a}^{*}\right|_{E}\right)$. Replacing $T$ by $T^{-1}$ yields $T^{-1}\left(\operatorname{ker}\left(\left.T_{a}^{*}\right|_{E}\right)\right) \subseteq \operatorname{ker}\left(T_{a}\right)$.

(c): Apply (b) to $T=T_{l}+T_{a}$.

\section{3。 Bogølind}

For each $T \in \mathscr{T}(E)$ there exists a (unique) ${ }^{*}$-automorphism $\alpha_{T}$ on $\mathscr{W}(E)$ with

$$
\alpha_{T}(W(f))=W(T f) \quad \forall f \in E,
$$

which is called the Bogoliubov transformation associated with $T$. The dual $\nu_{T}:=$ $\alpha^{*}$ is an affine bijection on the state space $\mathscr{\&}$,

$$
\left\langle\nu_{T}(\omega) ; M\right\rangle=\left\langle\omega ; \alpha_{T}(M)\right\rangle \forall \omega \in \& \forall M \in \mathscr{W}(E) .
$$

Obviously, $\left(\nu_{T}\right)^{-1}=\nu_{T^{-1}}$, and $C_{\nu_{T}(\omega)}=C_{\omega} \circ T$. Since a symplectic $T \in \mathscr{T}(E)$ is real-linear and bijective, the state $\omega \in \mathcal{S}$ is regular, of class $\mathscr{C}^{m}$, analytic, entire-analytic, if and only if $\nu_{T}(\omega)$ is so, respectively. Obviously, $\operatorname{Var}\left(\nu_{T}(\omega) ; f\right)$ $=\operatorname{Var}(\omega ; T f), f \in E$.

By reasons which are elaborated in [26], the Bogoliubov transformation $\alpha_{T}$ respectively $\nu_{T}$ is called a squeezing transformation, if $T_{a} \neq 0$, or equivalently, if $T$ is non-unitary.

Special Bogoliubov transformations are the gauge transformations of the first kind, $T=z \mathrm{H}_{E}$ with $z \in T:=\{z \in \mathbb{C}|| z \mid=1\}$. We write $\nu_{z}$ for the associated gauge symmetry transformations on $\&$ in the Schrödinger picture. An immediate consequence is 
Observation 2.5. Let $T \in \mathscr{T}(E)$. We have $T_{a} \neq 0$, if and only if $\nu_{z} \circ \nu_{T} \neq \nu_{T} \circ$ $\nu_{z}$ for some $z \in T$, that is, if some gauge transformations do not commute with the Bogoliubov transformation associated with $T$.

Proof. For the proof notice, $W(f)=W(g)$, if and only if $f=g$.

\section{§3. Transformations of Classical States}

In quantum optics a classical state of the photon field is defined by the positivity of the measure in the so-called $P$-representation (of the characteristic function). This gives the normally ordered expectations the form of a classical mixture over pure coherent states, tacitly associated with the Fock representation. We generalize: Let $\mathbf{P}(E)$ be the convex set of all positivedefinite functions $P: E \rightarrow \mathbb{C}$ with $P(0)=1$ on the additive group $(E,+)[8]$. It holds

$$
|P(f)| \leq P(0)=1 \quad \forall f \in E \quad \forall P \in \mathbb{P}(E) .
$$

If, e.g., $P(f):=\exp \{-u(f, f)\}$ for a symmetric real-bilinear form $u: E \times E \rightarrow \mathbb{R}$, then $P \in \mathbb{P}(E)$, if and only if $u$ is positive, that is $u(f, f) \geq 0 \forall f \in E$.

Definition 3.1 (Classical States). A state $\omega \in \&$ is called classical, if its nomally ordered characteristic function $P_{\omega}$ is a positive-definite function on the additive group $E$, that is, if there exists a $P_{\omega} \in \mathbb{P}(E)$ with $C_{\omega}=C_{\mathrm{vac}} P_{\omega}$, where $C_{\mathrm{vac}}$ is the characteristic function (2.1) of the vacuum state $\omega_{\mathrm{vac}} \in \mathscr{\&}$. The set of all classical states on $\mathbb{W}(E)$ is denoted by $\oiint_{c l}$.

Observation 3.2. Since the product of two positive-definite kernels gives again a positive-definite kernel, we have $C_{\mathrm{vac}} P \in \mathbf{C}(E)$ for each $P \in \mathbb{P}(E),[8],[20]$, [21], [33]. Thus, by Lemma 2.1 there exists for each $P \in \mathbb{P}(E)$ a unique state $\omega \in$ \& with $C_{\omega}=C_{\mathrm{vac}} P$, that is, with $P=P_{\omega}$. This defines a one-to-one correspondence between $\mathscr{\ell}_{c l}$ and $\mathrm{P}(E)$.

Indeed, the above definition of a classical state is a generalization of the positive $P$-representation, which is obtained by harmonic analysis. Consider the additive abelian group $E$ with discrete topology and its character group $\widehat{E}$, which is compact in the $\triangle$-topology (the topology of pointwise convergence: $\lim _{\imath} \chi_{\imath}=\chi$ in $\widehat{E}$, if and only if $\left.\lim _{\imath} \chi_{\imath}(f)=\chi(f) \forall f \in E\right)$. For each $\chi \in \widehat{E}$ there is a $*_{-}$automorphism $\gamma_{\chi}$ on $W(E)$ with $\gamma_{\chi}(W(f))=\chi(f) W(f) \forall f \in E$, a gange transformation of the second kind. Let

$$
\varphi_{\chi}:=\omega_{\mathrm{vac}}{ }^{\circ} \gamma_{\chi} \in \partial_{e} \& \text {, that is } C_{\varphi_{\chi}}=C_{\mathrm{vac}} \chi,
$$

which is pure, since $\omega_{\text {vac }}$ is so. Because of $\widehat{E} \subset \mathbf{P}(E)$, it holds $\varphi_{\chi} \in \bigotimes_{c l}$ with $P_{\varphi_{\chi}}=$ $\chi$. Let $\mathbf{M}_{+}^{1}(\widehat{E})$ be the (positive) probability measures on $\widehat{E}$, which constitutes 
also the weak ${ }^{*}$-compact state space of the $\mathrm{C}^{*}$-algebra of continuous functions on $\widehat{E}$. Via the affine one-to-one correspondence between $\mathbb{P}(E)$ and $\mathbb{M}_{+}^{1}(\widehat{E})$, which is given by $P(f)=\int_{\hat{E} \chi}(f) \mathrm{d} \mu(\chi) \forall f \in E$ (Bochner's theorem [13]), one proves:

Proposition 3.ふ. There exists an affine homeomorphism

$$
\Upsilon: \mathscr{S}_{c l} \rightarrow \mathbb{M}_{+}^{1}(\widehat{E}), \omega \mapsto \mu_{\omega} \text { with } \omega=\int_{\widehat{E}} \varphi_{\chi} \mathrm{d} \mu_{\omega}(\chi) .
$$

This especially implies for each $\omega \in \mathscr{\Theta}_{c l}$,

$$
P_{\omega}(f)=\int_{\hat{E}} \chi(f) \mathrm{d} \mu_{\omega}(\chi) \quad \forall f \in E .
$$

Since $\mathbb{M}_{+}^{1}(\widehat{E})$ is a Bauer simplex, so is $\mathscr{S}_{c l}$ (equipped with the weak*-topology), the extreme boundary $\partial_{e} \oiint_{c l}$ of which consists just of the pure, classical states

$$
\mathscr{\Theta}_{c l} \cap \partial_{e} \Theta=\partial_{e} \bigotimes_{c l}=\left\{\varphi_{\chi} \in \& \mid \chi \in \widehat{E}\right\} .
$$

The unique extremal decomposition in Proposition 3.3 illustrates the classical structure of the classical states $\mathscr{\ell}_{c l}$ introduced in Definition 3.1 [33].

If $\chi(f):=\exp \{i \sqrt{2} \operatorname{Re}(G(f))\}, f \in E$, with respect to a (complex-) linear form $G: E \rightarrow \mathbb{C}$, then the associated state $\varphi_{\chi} \in \partial_{e} \oiint_{c l}$ is quantum optically coherent of order $\infty$ according to Definition 5.1 below. For bounded $G$ (with respect to the norm of $E$ ) the state $\varphi_{\chi}$ is a coherent Glauber state. which is realizable by a so-called Glauber vector in Fock space [9], [17], [20]. This suggests the notion of a generalized Glauber coherent state for $\varphi_{\chi}$ also if $\chi \in \widehat{E}$ does not arise from a linear form, cf. [26].

We now investigate the behaviour of the classical states $\mathscr{\ell}_{c l}$ with respect to Bogoliubov transformations.

Lemma 3.4. For $T \in \mathscr{T}(E)$ and $\omega \in \mathscr{S}_{c l}$ the following assertions are equivalent:

(i) $\nu_{T}(\omega) \in \mathscr{\bigotimes}_{c l}$.

(ii) $N_{F}^{T} P_{\omega} \in \mathbb{P}(E)$, where $N_{F}^{T}$ is given by

$$
f \in E \mapsto N_{F}^{T}(f):=\exp \left\{\frac{1}{4}\left(\left\|T^{-1} f\right\|^{2}-\|f\|^{2}\right)\right\} .
$$

Proof. Since $C_{\nu_{T}(\omega)}=C_{\omega} \circ T=C_{\mathrm{vac}}\left(N_{F}^{T} \circ T\right)\left(P_{\omega} \circ T\right)$, it follows that $\nu_{T}(\omega) \in$ $\mathscr{S}_{c l}$, if and only if $\left(N_{F}^{T} \circ T\right)\left(P_{\omega} \circ T\right) \in \mathrm{P}(E)$. But the map $P \mapsto P \circ T$ is a bijection on $\mathbf{P}(E)$. Thus $\left(N_{F}^{T} \circ T\right)\left(P_{\omega} \circ T\right) \in \mathbb{P}(E)$, if and only if $N_{F}^{T} P_{\omega} \in \mathbb{P}(E)$.

Theorem 3.5. Let $T \in \mathcal{T}(E)$. Then we have:

(a) The following conditions are equivalent:

(i) $T_{a} \neq 0$;

(ii) $\nu_{T}\left(\omega_{\mathrm{vac}}\right) \notin \oiint_{c l}$, i.e., the transformed vacuum is non-classical; 
(iii) there exists an $\omega \in \partial_{e} \oiint_{c l}$ with $\nu_{T}(\omega) \notin \oiint_{c l}$;

(iv) there exists an $\omega \in \mathscr{S}_{c l}$ with $\nu_{T}(\omega) \notin \mathscr{S}_{c l}$.

(b) For $T_{a} \neq 0$ it holds $\nu_{T}\left(\partial_{e} \oiint_{c l}\right) \cap \mathscr{\bigotimes}_{c l}=\emptyset$, but $\nu_{T}\left(\&_{c l}\right) \cap \oiint_{c l} \neq \emptyset$.

(c) For $T_{a}=0$ it holds $\nu_{T}\left(\partial_{e} \varkappa_{c l}\right)=\partial_{e} \varkappa_{c l}$, and $\nu_{T}\left(\Theta_{c l}\right)=\oiint_{c l}$.

Proof. $\quad\left(\right.$ a) $(\mathrm{i}) \Rightarrow$ (a) (ii): For the vacuum state $\omega_{\text {vac }}$ it holds $P_{\omega_{\mathrm{vac}}}(f)=1 \forall f$ $\in E$. Thus with Lemma 3.4 we only have to show that $N_{F}^{T} \notin \mathbb{P}(E)$, where $N_{F}^{T}$ is defined in (3.3). But by Lemma 2.4 (b) there exists a $g \in E$ with $N_{F}^{T}(g)>N_{F}^{T}(0)$ $=1$, which contradicts (3.1). Hence $N_{F}^{T} \notin \mathbb{P}(E)$. (a) (ii) $\Rightarrow$ (a) (iii) is immediate. sirce $\omega_{\text {vac }}=\varphi_{x}$ with $\chi \equiv 1 \in \widehat{E}$. (a) (iii) $\Rightarrow$ (a) (iv) is trivial. (a) (iv) $\Rightarrow$ (a) (i): fissume $\overrightarrow{{ }_{c}}=0$. By Lemma 2.4 (a) $T$ is unitary. Hence $N_{F}^{T} \equiv 1$, and $\nu_{T}(\omega) \in \AA_{c l}$ by Lemma 3.4, a contradiction. The arguments here also prove (c). (b): Let $\chi$ $\in \widehat{E}$. Since $|\chi(f)|=1 \forall f \in E$ it holds $\left|N_{F}^{T}(g) \chi(g)\right|>\mathbb{1}$ for the above $g \in E$. Thus $N_{F}^{T} z^{\prime} \& F^{n}(E)$ by $(3,1)$. Now Lemma $3 . \Delta$ implies $\nu_{T}\left(\varphi_{\chi}\right) \notin \&_{c l}$. The rest follows from Erample 4.3 below.

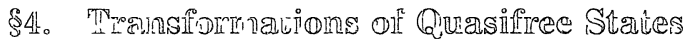

The gereral notion of a quasilree state, whose higher-order iruncated functionals vanish, was introducea in [34], [35] (cr. also [4] [14]). We do not give the original definition, but we state a result, which characterizes all quasifree states, [1], [2] [36], L30]. [15] (cf. also [3]).

Theolem 4.1 (Guarisifiree Staites)。 Let be $\ell: E \rightarrow \mathbb{R}$ a real-linear fom and $s:$ $\Sigma \times E \rightarrow \mathbb{R}$ a positive surnetric real-bilinear form satisfying

$$
\left.\lim \langle f \mid g\rangle\right|^{2} \leq_{s}(f, f)_{s}(g, g) \quad \forall f, g \in E .
$$

Then ihere exists a unique state $\omega \in \&$ with the characteristic function

$$
C_{\omega}(f):=\exp \left\{i \ell(f)-\frac{1}{4} s(f, f)\right\} \quad \forall f \in E .
$$

$\omega$ is uniasifree (especially entire-analytic), and satisfies

$$
\operatorname{Var}(\omega ; f)=\frac{1}{2} s(f, f),\left\langle\omega ; \overline{\mathbb{Q}}_{\omega}(f)\right\rangle=\ell(f), \forall f \in E .
$$

Moreover, every quasifree state on $\mathbb{W}(E)$ has the form (4.2) with s satisfying (4.1). The set of all quasifree states on $\mathbb{W}(E)$ is denoted by $\&_{q f}$.

We have $\omega \in \mathscr{\bigotimes}_{q f} \cap \mathscr{\bigotimes}_{c l}$, if and only if the associated symmetric real-bilinear form $t(f, g):=s(f, g)-\operatorname{Re}\langle f \mid g\rangle, f, g \in E$ is positive. In this case

$$
P_{\omega}(f)=\exp \left\{i \ell(f)-\frac{1}{4} t(f, f)\right\} \quad \forall f \in E
$$


for the corresponding $P_{\omega} \in \mathbf{P}(E)$.

$\omega \in \oiint_{q f}$ is gauge-invariant (that is, $\nu_{z}(\omega)=\omega \forall z \in T$ ), if and only if $t$ is a positive sesquilinear form on $E$ (anti-linear in the first factor, linear in the second factor) and $\ell=0$. In this case $\omega \in \mathscr{S}_{c l}$ with $P_{\omega}(f)=\exp \left\{-\frac{1}{4} t(f, f)\right\}$, and $t(f, g)=$ $2 \cdot\left\langle\omega ; a_{\omega}^{*}(g) a_{\omega}(f)\right\rangle \forall f, g \in E$.

Quasifree states (also called Gaussian states) play an important role in statistical physics, since, e.g., the thermodynamic equilibrium states (limiting Gibbs and KMS states) for the photons and those for the free Boson gas (with and without Bose-Einstein condensation) are quasifree, gauge-invariant, and thus classical states, [12], [3], [6], [28], [5], [11], [4].

Theorem 4.2。 Let $T \in \mathscr{T}(E)$. Then it holds:

(a) $\nu_{T}\left(\&_{q f}\right)=\oiint_{q f}$.

(b) Let $\omega \in \mathscr{\bigotimes}_{q f} \cap \mathscr{S}_{c l}$ with associated real-linear form $\ell$ and symmetric real-bilinear form $t$ from (4.4). We have the following equivalence:

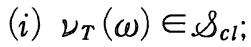

(ii) the symmetric real-bilinear form

$$
t_{T}: E \times E \rightarrow \mathbb{R},(f, g) \mapsto t(f, g)+\operatorname{Re}\langle f \mid g\rangle-\operatorname{Re}\left\langle T^{-1} f \mid T^{-1} g\right\rangle
$$

is positive.

Proof. (a): Let $\omega \in \mathscr{\bigotimes}_{q f}$ with associated $\ell$ and $s$ according to (4.2). Then

$$
C_{\nu_{T}(\omega)}(f)=C_{\omega}(T f)=\exp \left\{i \ell(T f)-\frac{1}{4} s(T f, T f)\right\}=\exp \left\{i \widehat{\ell}(f)-\frac{1}{4} \widehat{s}(f, f)\right\} \forall f \in E .
$$

But $\widehat{\ell}:=\ell \circ T$ is a real-linear form on $E$ and the positive symmetric real-bilinear form $\hat{s}(f, g):=s(T f, T g), f, g \in E$, satisfies (see the equations (2.6) and (4.1))

$$
\left.\left|\operatorname{Im}\langle f \mid g\rangle^{2}=\right| \operatorname{Im}\langle T f \mid T g\rangle\right|^{2} \leq_{s}(T f, T f)_{s}(T g, T g)=\hat{s}(f, f) \hat{s}(g, g) \quad \forall f, g \in E .
$$

Thus $\widehat{s}$ fulfills $(4.1)$, and consequently, $\nu_{T}(\omega) \in \mathscr{\bigotimes}_{q f}$ by the above Theorem. Up to now we have shown $\nu_{T}\left(\oiint_{q f}\right) \subseteq \oiint_{q f}$. The same argumentation for $T^{-1} \in \mathscr{T}(E)$ gives $\nu_{T^{-1}}\left(\&_{q f}\right) \subseteq \mathscr{\ell}_{q f}$. Now $\left(\nu_{T}\right)^{-1}=\nu_{T^{-1}}$ yields the result. $(\mathrm{b}): N_{F}^{T} P_{\omega} \in \mathbb{P}(E)$ is equivalent to (b) (ii). Now use Lemma 3.4.

Example 4. \&. Let $T \in \mathscr{T}(E)$, and define $t^{0}(f, g):=\operatorname{Re}\left\langle T^{-1} f \mid T^{-1} g\right\rangle, f, g \in E$. For an arbitrary positive symmetric real-bilinear form $\tilde{t}$ on $E$ let $t:=t^{0}+\tilde{t}$. Then $t$ and $t_{T}$ from equation (4.5) are positive.

For an arbitrary real-linear form $\ell: E \rightarrow \mathbb{R}$ we define the state $\omega \in \mathscr{\bigotimes}_{c l}$ by $C_{\omega}:=$ $C_{\mathrm{vac}} P_{\omega}$ with the positive-definite function $P_{\omega}(f):=\exp \left\{i \ell(f)-\frac{1}{4} t(f, f)\right\}, f \in E$. Then by the Theorems 4.1 and 4.2 it holds $\omega \in \oiint_{q f} \cap \oiint_{c l}$ and $\nu_{T}(\omega) \in \oiint_{q f} \cap \oiint_{c l}$. 


\section{§5. Transformations of Classical Coherent States}

A smearing procedure of Glauber's original factorization condition leads to the following operator algebraic formulation of quantum optical coherence [20], [21], [19], where the linear form $L: E \rightarrow \mathbf{C}$ replaces the coherence function (cf. the Introduction).

Definition 5.1 (Coherent States)。 Let $n \in \mathbb{N} \cup\{\infty\}$. An analytic state $\omega \in$ $\&$ is called coherent of $n^{-}$th order with respect to the (complex-) linear form $L: E \rightarrow \mathbb{C}$, if

$$
\left\langle\omega ; a_{\omega}^{*}\left(f_{1}\right) \cdots a_{\omega}^{*}\left(f_{m}\right) a_{\omega}\left(g_{1}\right) \cdots a_{\omega}\left(g_{m}\right)\right\rangle=L\left(f_{1}\right) \cdots L\left(f_{m}\right) \overline{L\left(g_{1}\right)} \cdots \overline{L\left(g_{m}\right)}
$$

for all $f_{1}, \ldots, f_{m}, g_{1}, \ldots, g_{m} \in E$ and each $m \in \mathbb{N}$ with $1 \leq m \leq n$. We denote by $\mathscr{\bigotimes}_{L}^{(n)}$ the convex set of all $n$-th order coherent states on $W(E)$ with respect to $L$.

Observation 5.2. For the linear form $L: E \rightarrow \mathbb{C}$ it holds:

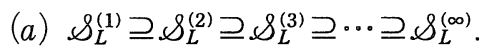

(b) $\mathscr{\bigotimes}_{z L}^{(n)}=\mathscr{\Theta}_{L}^{(n)}$ for every $z \in \mathbb{T}=\{z \in \mathbb{C}|| z \mid=1\}$ and all $n \in \mathbb{N} \cup\{\infty\}$.

(c) If $L=0$, then $\mathscr{S}_{0}^{(1)}=\oiint_{0}^{(2)}=\cdots=\oiint_{0}^{(\infty)}=\left\{\omega_{\mathrm{vac}}\right\}$.

Before summarizing some essential results concerning coherent states, we introduce two Bauer sub-simplices of $\varkappa_{c l}$. Let $b \mathbb{C}$ be the Bohr compactification of $\mathbb{C}[13]$. Each function $z \in \mathbb{C} \mapsto \xi_{\alpha}(z):=\exp \{i \sqrt{2} \operatorname{Re}(z \alpha)\}$ extends uniquely to a continuous function on $b \mathbb{C}$. Moreover, $\left\{\xi_{\alpha} \mid \alpha \in \mathbb{C}\right\}$ generates $\mathrm{C}^{*}$-algebraically the continuous functions on $b \mathbb{C}$ which are isomorphic to the almost periodic functions on $\mathbb{C}[13]$. Obviously, $f \in E \mapsto \chi_{z}^{L}(f):=\exp \{i \sqrt{2} \operatorname{Re}(z L(f))\}$ is an element of $\widehat{E}$. This defines the continuous embedding $\iota_{L}: b \mathbb{C} \rightarrow \widehat{E}, z \mapsto \chi_{z}^{L}$. Thus the torus $\iota_{L}(\mathbb{T})$ and $\iota_{L}(b \mathbb{C})$ are compact subsets of $\widehat{E}$, and $\iota_{L}(\mathbb{C})$ is an open subset of $\widehat{E}$ (the canonical embedding of $\mathbb{C}$ into $b \mathbb{C}$ is an open map [13]). The associated embedding of the measures $\widetilde{\iota_{L}}: \mathbb{M}_{+}^{1}(b \mathbb{C}) \rightarrow \mathbb{M}_{+}^{1}(\widehat{E})$ is given as $\widetilde{\iota_{L}}(\mu)=\mu \circ \iota_{L}^{-1}$. Obviously it holds

$$
\iota_{L}(\mathbb{T}) \subset \iota_{L}(\mathbb{C}) \subset \iota_{L}(b \mathbb{C}) \subseteq \widehat{E}, \widetilde{\iota_{L}}\left(\mathbb{M}_{+}^{1}(\mathbb{T})\right) \subset \widetilde{c_{L}}\left(\mathbb{M}_{+}^{1}(\mathbb{C})\right) \subset \widetilde{\iota_{L}}\left(\mathbb{M}_{+}^{1}(b \mathbb{C})\right) \subseteq \mathbb{M}_{+}^{1}(\widehat{E}) .
$$

$\left(c_{L}(b \mathbb{C})=\widehat{E}\right.$ is equivalent to $\operatorname{dim}(E)=1$.) $\mathbf{M}_{+}^{1}(\mathbb{T}), \mathbf{M}_{+}^{1}(b \mathbb{C})$, and $\mathbf{M}_{+}^{1}(\widehat{E})$ are Bauer simplices, but $\mathbb{M}_{+}^{1}(\mathbb{C})$ is not so, since $\mathbb{C}$ is not compact. By restricting $\Upsilon^{-1}$ of Proposition 3.3 we obtain convex subsets of $\mathscr{S}_{c l}$, especially the Bauer simplices $\left(\gamma^{-1} \circ \widetilde{\iota_{L}}\right)\left(\mathbb{M}_{+}^{1}(\mathbf{T})\right)$ and $\mathscr{\ell}_{c l}^{L}:=\left(\boldsymbol{\gamma}^{-1} \circ \widetilde{\iota_{L}}\right)\left(\mathbf{M}_{+}^{1}(b \mathbb{C})\right)$. Let $\mu_{\omega}^{L} \in \mathbf{M}_{+}^{1}(b \mathbf{C})$ be the measure uniquely associated with $\omega \in \mathscr{\ell}_{c l}^{L}$ via $\Upsilon^{-1} \circ \widetilde{\iota_{L}}$. Then $\mu_{\omega}=\widetilde{\iota_{L}}\left(\mu_{\omega}^{L}\right) \in$ $M_{+}^{1}(\widehat{E})$, and for the positive-definite function $P_{\omega} \in \mathbf{P}(E)$ from (3.2) we obtain 
the integral decomposition

$$
P_{\omega}(f)=\int_{\bar{E}} \chi(f) \mathrm{d} \mu_{\omega}(\chi)=\int_{b \mathrm{C}} \exp \{i \sqrt{2} \operatorname{Re}(z L(f))\} \mathrm{d} \mu_{\omega}^{L}(z) \quad \forall f \in E
$$

for every $\omega \in \mathscr{S}_{c l}^{L}$. For $\mu_{\omega}^{L} \in \mathbb{M}_{+}^{1}(\mathbb{C})$ we define the moments (if the integrals exist)

$$
c_{\omega}^{L}(k, l):=\int_{\mathbb{C}} z^{k} \bar{z}^{l} \mathrm{~d} \mu_{\omega}^{L}(z), 0 \leq k, l<\infty
$$

Obviously, $c_{\omega}^{L}(l, k)=\overline{c_{\omega}^{L}(k, l)}$. The measure $\mu_{\omega}^{L} \in \mathbb{M}_{+}^{1}(\mathbb{C})$ is called (entire-) analytic, if $\int_{\mathbb{C}} \exp \{\delta|z|\} \mathrm{d} \mu_{\omega}^{L}(z)<\infty$ for some (all) $\delta>0$.

In the subsequent theorem we compile some structural results on coherent states, extracted from [20], [21], [22], [17].

Theorem 5.8. Let $L: E \rightarrow \mathbb{C}$ be a non-zero linear form. It holds (a) $\omega_{\mathrm{vac}} \in \Theta_{c l}^{L}$, and $\mu_{\omega_{\mathrm{vac}}}^{L} \in \mathbb{M}_{+}^{1}(\mathbb{C})$ is the point measure at the origin $z=0$.

(b) $\omega \in \mathscr{\bigotimes}_{c l}^{L}$ is regular, if and only if $\mu_{\omega}^{L} \in \mathbb{M}_{+}^{1}(\mathbb{C})$, ihat is, $\mu_{\omega}^{L}(b \mathbb{C} \backslash \mathbb{C})=0$. Let

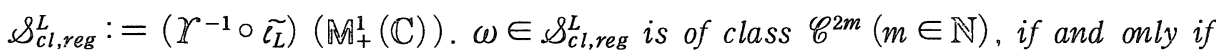
$c_{\omega}^{L}(m, m)<\infty$, in which case for all $f_{1}, \ldots, f_{k}, g_{1}, \ldots, g_{l} \in E$ and each $0 \leq k, l \leq m$ :

$$
\left\langle\omega ; a_{\omega}^{*}\left(f_{1}\right) \cdots a_{\omega}^{*}\left(f_{k}\right) a_{\omega}\left(g_{1}\right) \cdots a_{\omega}\left(g_{l}\right)\right\rangle=c_{\omega}^{L}(k, l) L\left(f_{1}\right) \cdots L\left(f_{k}\right) \overline{L\left(g_{1}\right)} \cdots \overline{L\left(g_{l}\right)} .
$$

$\omega \in \mathscr{S}_{c l, r e g}^{L}$ is (entire-) analytic, if and only if $\mu_{\omega}^{L} \in \mathbb{M}_{+}^{1}(\mathbb{C})$ is so.

(c) $\mathscr{S}_{L}^{(1)} \cap \mathscr{S}_{c l} \subset \mathscr{\ell}_{c l, \text { reg. }}^{L} \omega \in \mathscr{\bigotimes}_{c l, \text { reg }}^{L}$ is an element of $\mathscr{\bigotimes}_{L}^{(1)} \cap \mathscr{S}_{c l}$, if and only if $\mu_{\omega}^{L}$ is analytic and $c_{\omega}^{L}(1,1)=1$.

(d) $\mathscr{S}_{L}^{(n)} \cap \mathscr{S}_{c l}=\mathscr{S}_{L}^{(\infty)} \cap \mathscr{S}_{c l}=\left(\mathscr{Y}^{-1} \circ \widetilde{\iota_{L}}\right)\left(\mathbb{M}_{+}^{1}(\mathbb{T})\right)$ (and hence is a Bauer simplex) for all $n \geq 2$. $\omega \in \mathscr{\bigotimes}_{c l, r e g}^{L}$ is an element of $\mathscr{\bigotimes}_{L}^{(\infty)} \cap \mathscr{\bigotimes}_{c l}$, if and only if $c_{\omega}^{L}(1,1)=1=$ $c_{\omega}^{L}(2,2)$.

(e) $\oiint_{L}^{(1)} \subset \oiint_{c l}$, if and only if $L$ is unbounded (with respect to the norm of $E$ ). That is, only for bounded $L$ there exist non-classical states $\omega \in \mathscr{S}_{L}^{(n)}, n \in \mathbb{N} \cup\{\infty\}$, some examples of which are found in [18] and [23] for $n=\infty$.

(f) Let $\omega \in \mathscr{\bigotimes}_{L}^{(1)}$, or let $\omega \in \mathscr{S}_{\text {cl,reg }}^{L} \backslash\left\{\omega_{\mathrm{vac}}\right\}$. Then $\omega$ is normal to the Fock representation, if and only if $L$ is bounded. Especially, each non-classical coherent state of any order is normal to the Fock representation.

( $g$ ) By the parts $(d)$ and $(e)$ it holds

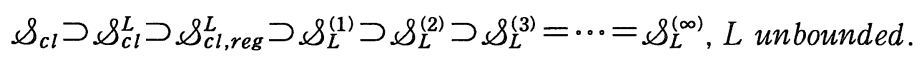

For bounded $L$ the inclusions in Observation 5.2 (a) are proper, and one has

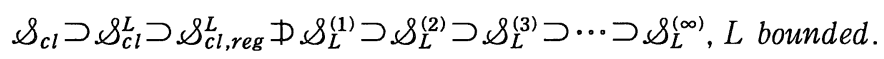


Corollary 5.4. Let $L$ be a non-zero linear form on E. It holds

(a) $\mathscr{\ell}_{c l}^{L}=\Theta_{c l}^{v L}$ and $\mathscr{\Theta}_{c l, r e g}^{L}=\oiint_{c l, r e g}^{v L}$ for each non-zero $v \in \mathbb{C}$.

(b) $c_{\omega}^{L}(k, l)=v^{k} \bar{v}^{l} c_{\omega}^{\nu L}(k, l)$ for all $0 \leq k, l<\infty$ and each non-zero $v \in \mathbb{C}$.

(c) If $\omega \in \mathscr{\ell}_{c l}^{L}$ is analytic, then $\omega \in \mathscr{\bigotimes}_{\lambda L}^{(1)}$ where $\lambda:=\sqrt{c_{\omega}^{L}(1,1)}$.

(d) If $\omega \in \mathcal{\&}_{c l}^{L}$ is of class $\mathscr{C}^{2}$, then

$$
\begin{aligned}
\left\langle\omega ; \bar{\Phi}_{\omega}(f)\right\rangle= & \sqrt{2} \operatorname{Re}\left(L(f) c_{\omega}^{L}(1,0)\right), \\
\operatorname{Var}(\omega ; f)= & \operatorname{Re}\left(L(f)^{2}\left[c_{\omega}^{L}(2,0)-c_{\omega}^{L}(1,0)^{2}\right]\right) \\
& \quad+|L(f)|^{2}\left[c_{\omega}^{L}(1,1)-\left|c_{\omega}^{L}(1,0)\right|^{2}\right]+\frac{1}{2}\|f\|^{2} .
\end{aligned}
$$

Proof. $\mu_{\omega}^{L} \circ v=\mu_{\omega}^{v L}$ and $\int_{\mathbb{C}} f(z) \mathrm{d} \mu_{\omega}^{L}(z)=\int_{\mathbb{C}} f(v z) \mathrm{d} \mu_{\omega}^{v L}(z)$. For (d) use the CCR and Theorem $5.3(\mathrm{~b})$.

Observe that (5.3) gives a non-vanishing field expectation only if $c_{\omega}^{L}(1,0) \neq 0$ and that the field fluctuations in (5.4) decompose into the quantum mechanical vacuum part $\operatorname{Var}\left(\omega_{\mathrm{vac}} ; f\right)=\frac{1}{2}\|f\|^{2}$ and the remaining classical part (for more details to the decomposition of the fluctuations we refer to [26]).

We now investigate the behaviour of the Bauer simplex $\oiint_{c l}^{L}$ with respect to Bogoliubov transformations.

Thle@rem 5.5. Assume $T \in \mathscr{T}(E)$ and let $L: E \rightarrow \mathbb{C}$ be a non-zero linear form. Then the following assertions are valid:

(a) Let $T_{a}=0$ (or equivalently, $T$ linear resp. unitary by Lemma 2.4). Then $f \in E \mapsto$ $L(T f)$ is a non-zero linear form. It holds $\nu_{T}\left(\Theta_{c l}^{L}\right)=\mathscr{\ell}_{c l}^{L T}, \nu_{T}\left(\oiint_{c l, r e g}^{L}\right)=\mathscr{\ell}_{c l, \text { reg }}^{L T}$, and

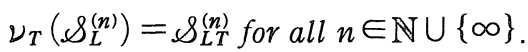

(b) Let $\left.T_{a}^{*}\right|_{\mathrm{ker}(L)} \neq 0$. Then $\nu_{T}\left(\mathscr{\ell}_{c l}^{L}\right) \cap \mathscr{\bigotimes}_{c l}=\emptyset$. Moreover, for each analytic, and thus

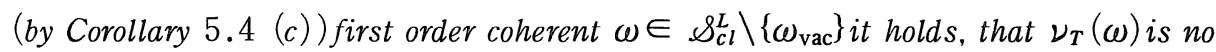
longer coherent in any order with respect to any linear form $G: E \rightarrow \mathbb{C}$.

(c) Let $\mathrm{T}_{a} \neq 0$ and $\operatorname{ker}(L) \subseteq \operatorname{ker}\left(T_{a}^{*}\right)$ (or equivalently, $\left.\left.T_{a}^{*}\right|_{\operatorname{ker}(L)}=0\right)$. Then $T_{l}, T_{a}$, and $L$ are bounded, and we have the case of one-mode squeezing. That is, there exist unique normalized $h_{L}, h_{Q} \in E$ and a unique phase $v_{L} \in T$ such that

$\circ L(f)=v_{L}\|L\|\left\langle h_{L} \mid f\right\rangle$ for all $f \in E$;

- $T_{a} f=\left\|T_{a}\right\|\left\langle f \mid h_{Q}\right\rangle h_{L}$, resp. $T_{a}^{*} f=\left\|T_{a}\right\|\left\langle f \mid h_{L}\right\rangle h_{Q}$, for all $f \in E$;

- $\bar{T}_{l}$ is a bijective isometry from $h_{Q}{ }^{\perp}$ onto $h_{L}{ }^{\perp}$;

- $T_{l} h_{Q}=\sqrt{1+\left\|T_{a}\right\|^{2}} h_{L}$, and $T_{l}^{*} h_{L}=\sqrt{1+\left\|T_{a}\right\|^{2}} h_{Q}$.

(d) Let $T_{a} \neq 0$. Then $\nu_{T}\left(\omega_{\mathrm{vac}}\right)$ is coherent in first order with respect to some linear form $G: E \rightarrow \mathbb{C}$, if and only if $\operatorname{dim}\left(T_{a}(E)\right)=1$, that is, if and only if we have one-mode squeezing (observe that $\omega_{\mathrm{vac}} \in \oiint_{c l}^{L}$ for every linear form $L: E \rightarrow \mathbb{C}$ by Theorem $5.3(a))$. But $\nu_{T}\left(\omega_{\mathrm{vac}}\right)$ is always non-classical for $T_{a} \neq 0$ (by Theorem 3.5 
$(b)$, since $\left.\omega_{\mathrm{vac}} \in \partial_{e} \bigotimes_{c l}\right)$.

Proof. (a) is immediate. (b): Let $\omega \in \mathscr{S}_{c l}^{L}$. We use Lemma 3.4 to show that $\nu_{T}(\omega) \notin \mathscr{S}_{c l}$. By Lemma 2.4 (b) there exists a $g \in \operatorname{ker}(L)$ with $N_{F}^{T}(g)>N_{F}^{T}(0)=$ 1 , where $N_{F}^{T}$ is from (3.3). Since $g \in \operatorname{ker}(L)$, it follows from (5.1) that $P_{\omega}(g)=$ 1. Thus $N_{F}^{T}(g) P_{\omega}(g)>1$, which with (3.1) yields $N_{F}^{T} P_{\omega} \notin \mathbb{P}(E)$. Consequently, $\nu_{T}(\omega) \notin \oiint_{c l}$ by Lemma 3.4 .

Now we prove that $\nu_{T}(\omega)$ is no longer coherent for each analytic $\omega \in \oiint_{c l}^{L}$. By Theorem 5.3(b) $\omega \in \mathscr{O}_{c l}^{L}$ analytic is equivalent to $\int_{\mathbf{C}} \exp \{\delta|z|\} \mathrm{d} \mu_{\omega}^{L}(z)<\infty$ for some $\delta>0$. Thus for each $f \in E$ there exists a neighbourhood $U_{f}=\overline{U_{f}} \subseteq \mathbb{C}$ of the origin such that the function

$$
\begin{aligned}
N_{\nu_{T}(\omega)} & (u, v ; f) \\
:= & \exp \left\{-\frac{1}{4}\left[2 u v\left\|T_{a} f\right\|^{2}+v^{2}\left\langle T_{l} f \mid T_{a} f\right\rangle+u^{2}\left\langle T_{a} f \mid T_{l} f\right\rangle\right]\right\} \\
& \times \int_{\mathbb{C}} \exp \left\{\frac{i}{\sqrt{2}}\left(u\left[z L\left(T_{l} f\right)+\bar{z} \overline{L\left(T_{a} f\right)}\right]+v\left[\overline{z L}\left(T_{l} f\right)+z L\left(T_{a} f\right)\right]\right)\right\} \mathrm{d} \mu_{\omega}^{L}(z)
\end{aligned}
$$

is analytic in the arguments $(u, v) \in U_{f} \times U_{f} \subseteq \mathbb{C}^{2}$. With (2.12) one checks the relation $C_{\nu_{T}(\omega)}(u f)=C_{\mathrm{vac}}(u f) N_{\nu_{T}(\omega)}(u, \bar{u} ; f) \forall u \in U_{f}$ and each $f \in E$, which identifies $P_{\nu_{T}(\omega)}(f)=N_{\nu_{T}(\omega)}(1,1 ; f)$ as the normally ordered characteristic function for $\nu_{T}(\omega)$ (which is positive-definite, if $\nu_{T}(\omega)$ is classical) according to Definition (2.5) and Lemma 2.2. Applying equation (2.4) for $k=l=1$ to $\nu_{T}(\omega)$ yields

$$
\begin{aligned}
\left\langle\nu_{T}(\omega) ; a^{*}(f) a(f)\right\rangle & =-\left.2 \frac{\partial^{2} N_{\nu T(\omega)}(u, v ; f)}{\partial u \partial v}\right|_{u=v=0} \\
& =\left\|T_{a} f\right\|^{2}+\int_{\mathbf{C}}\left|z L\left(T_{l} f\right)+\bar{z} \overline{L\left(T_{a} f\right)}\right|^{2} \mathrm{~d} \mu_{\omega}^{L}(z) \quad \forall f \in E .
\end{aligned}
$$

Let us from now on suppose that $\nu_{T}(\omega)$ is coherent, that is, $\nu_{T}(\omega) \in \mathscr{\ell}_{G}^{(1)}$ for some linear form $G: E \rightarrow \mathbb{C}$. But $\nu_{T}(\omega) \notin \oiint_{c l}$ implies $G$ to be bounded by

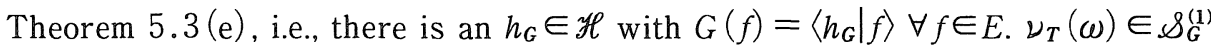
means $\left\langle\nu_{T}(\omega) ; a^{*}(f) a(f)\right\rangle=|G(f)|^{2}$. The comparison with (5.5) gives $\left\|T_{a} f\right\|^{2} \leq$ $|G(f)|^{2} \leq\left\|h_{G}\right\|^{2}\|f\|^{2} \forall f \in E$, which implies $T_{a}$ to be bounded and $\operatorname{ker}\left(T_{a}\right) \subseteq h_{G}{ }^{\perp}=$ $\operatorname{ker}(G)$. From $T_{a} \neq 0$ it follows $h_{G} \neq 0$ and thus $T_{a} f=\left\langle f \mid h_{G}\right\rangle h_{0} \forall f \in E$ with some $0 \neq h_{0} \in \mathscr{H}$. The boundedness of $T_{l}$, and thus of $T$, follows from $T_{l}^{*} T_{l}=T_{a}^{*} T_{a}+\mathbb{1}_{E}$ (eq. (2.9)). We continue $T_{l}, T_{a}$, and $T$ to all of $\mathscr{H}$ without changing the notation. The adjoint relation $T_{a}^{*} f=\left\langle f \mid h_{0}\right\rangle h_{G} \forall f \in \mathscr{H}, T_{a}(E) \subseteq E$, and $T_{a}^{*}(E) \subseteq E$ in fact give $h_{0}, h_{G} \in E$.

Specializing to the point measure at the origin, that is, $\omega=\omega_{\mathrm{vac}}$, we have

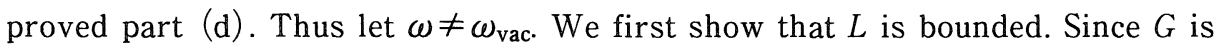
bounded, it follows from Theorem $5.3(\mathrm{f})$ that $\nu_{T}(\omega)$ is normal to the Fock 
representation $\Pi_{F}$ of $\mathscr{W}(E)$. But $T_{a}^{*}$ is an anti-linear Hilbert-Schmidt operator, and thus the Bogoliubov transformation $\alpha_{T^{-1}}$ on $\mathscr{W}(E)$ is unitarily implementable in $\Pi_{F}$ by [24]. Consequently, $\omega=\nu_{T^{-1}}\left(\nu_{T}(\omega)\right) \in \oiint_{c l, r e g}^{L}$ is normal to $\Pi_{F}$, too, which by Theorem $5.3(\mathrm{f})$ implies $L$ to be bounded. Let $L(f)=\left\langle h_{L} \mid f\right\rangle$ $\forall f \in \mathscr{H}$ with some $0 \neq h_{L} \in \mathscr{H}$. Then (5.5) leads to

$$
0=\left|\left\langle h_{G} \mid f\right\rangle\right|^{2}=\left\langle\nu_{T}(\omega) ; a^{*}(f) a(f)\right\rangle=\mid\left\langle h_{L}\left|T_{l} f\right|^{2} c_{\omega}^{L}(1,1) \quad \forall f \in \operatorname{ker}\left(T_{a}\right)=h_{G}{ }^{\perp},\right.
$$

where $c_{\omega}^{L}(1,1)>0$ since $\omega \neq \omega_{\text {vac. Thus }}\left\langle h_{L} \mid T_{l} f\right\rangle=0 \forall f \in \operatorname{ker}\left(T_{a}\right)$. But Lemma 2.4 (a) and equation (2.11) imply that $T$ and thus $T_{l}$ are bijective isometries from $\operatorname{ker}\left(T_{a}\right)=h_{G}{ }^{\perp}$ onto $\operatorname{ker}\left(T_{a}^{*}\right)=h_{0}{ }^{\perp}$. Hence there exists an $0 \neq \alpha \in \mathbb{C}$ with $h_{L}$ $=\alpha h_{0}$, implying $\operatorname{ker}\left(T_{a}^{*}\right)=\operatorname{ker}(L)$. This contradicts $\left.T_{a}^{*}\right|_{\operatorname{ker}(L)} \neq 0$, and thus our assumption of a first order coherent $\nu_{T}(\omega)$ is wrong.

(c): Since $L$ is a non-zero linear form, we have $\operatorname{dim}(E / \operatorname{ker}(L))=1$. Because of $\operatorname{ker}(L) \subseteq \operatorname{ker}\left(T_{a}^{*}\right), T_{a}^{*}$ defines the canonical map $\widetilde{T}_{a}^{*}: E / \operatorname{ker}(L) \rightarrow E$ with $\widetilde{T}_{a}^{*}(\widetilde{f}):=T_{a}^{*} f \forall f \in E$, where $f \mapsto \widetilde{f}$ is the canonical embedding from $E$ into the quotient $E / \operatorname{ker}(L)$. Since $T_{a} \neq 0$ implies $\left.T_{a}^{*}\right|_{E} \neq 0$, we have $\widetilde{T}_{a}^{*} \neq 0$. Hence there exists an $f_{0} \in E$ with $L\left(f_{0}\right)=1$ such that $0 \neq g_{0}:=T_{a}^{*} f_{0}=\widetilde{T}_{a}^{*}\left(\widetilde{f}_{0}\right) \in E$, that is, $\widetilde{f}_{0} \neq 0$. It holds $\tilde{f}=L(f) \tilde{f}_{0} \forall f \in E$, and thus it follows

$$
T_{a}^{*} f=\widetilde{T}_{a}^{*}(\widetilde{f})=\overline{L(f)} \widetilde{T}_{a}^{*}\left(\tilde{f}_{0}\right)=\overline{L(f)} g_{0} \quad \forall f \in E .
$$

Consequently, the linear form

$$
f \in E \mapsto L(f)=\left\|g_{0}\right\|^{-2}\left\langle T_{a}^{*} f \mid g_{0}\right\rangle=\left\|g_{0}\right\|^{-2}\left\langle T_{a} g_{0} \mid f\right\rangle
$$

is continuous. (5.6) then implies $T_{a}$ to be bounded, $\left\|T_{a}\right\|=\left\|T_{a}^{*}\right\|=\|L\|\left\|g_{0}\right\|$. The boundedness of $T_{l}$, and thus of $T$, follows from $T_{l}^{*} T_{l}=T_{a}^{*} T_{a}+\mathbb{H}_{E}$ (eq. (2.9)). We do not change the notation when continuing $T_{l}, T_{a}, L, T$, and $T^{-1}$ to all of $\mathscr{H}$. Now define $h_{L}^{\prime}$ by $L(f)=:\|L\|\left\langle h_{L}^{\prime} \mid f\right\rangle$, and set $h_{Q}^{\prime}:=\left\|g_{0}\right\|^{-1} g_{0} \in E$. Then (5.6) rewrites as $T_{a}^{*} f=\left\|T_{a}\right\|\left\langle f \mid h_{L}^{\prime}\right\rangle h_{Q}^{\prime}$, which gives the adjoint $T_{a} f=\left\|T_{a}\right\|\left\langle f \mid h_{Q}^{\prime}\right\rangle h_{L}^{\prime}$ for all $f \in E$. Especially it follows that $h_{L}^{\prime} \in E$, since $T_{a}(E) \subseteq E$. Lemma 2.4 (a) and equation (2.11) now imply that $T$ and thus $T_{l}$ are bijective isometries from $\operatorname{ker}\left(T_{a}\right)=h_{Q}^{\prime}{ }^{\perp}$ onto $\operatorname{ker}(L)=\operatorname{ker}\left(T_{a}^{*}\right)=h_{L}^{\prime}{ }^{\perp}$. Let us now determine the action of $T_{l}$ resp. $T_{l}^{*}$ on $h_{Q}^{\prime}$ resp. $h_{L}^{\prime}$. With $h_{Q}^{\prime}=\left\|T_{a}\right\|^{-1} T_{a}^{*} h_{L}^{\prime}$ and (2.10) we calculate

$$
\left\langle T_{l} h_{Q}^{\prime} \mid f\right\rangle=\left\|T_{a}\right\|^{-1}\left\langle T_{l} T_{a}^{*} h_{L}^{\prime} \mid f\right\rangle=\left\|T_{a}\right\|^{-1}\left\langle T_{a} T_{l}^{*} h_{L}^{\prime} \mid f\right\rangle=\left\|T_{a}\right\|^{-1}\left\langle T_{a}^{*} f \mid T_{l}^{*} h_{L}^{\prime}\right\rangle,
$$

from which follows $T_{l} h_{Q}^{\prime} \perp \operatorname{ker}\left(T_{a}^{*}\right)=h_{L}^{\prime}$, that is, $T_{l}^{*} h_{Q}^{\prime}=\gamma h_{L}^{\prime}$ for some $\gamma \in \mathbb{C}$. A similar argumentation leads to $T_{l}^{*} h_{L}^{\prime}=\varepsilon h_{Q}^{\prime}$ for some $\varepsilon \in \mathbb{C}$. $T_{l}^{*} T_{l} h_{Q}^{\prime}=\left(1+T_{a}^{*} T_{a}\right) h_{Q}^{\prime}$ $=\left(1+\left\|T_{a}\right\|^{2}\right) h_{Q}^{\prime}$ and $\left\langle T_{l} h_{Q}^{\prime} \mid h_{L}^{\prime}\right\rangle=\left\langle h_{Q}^{\prime} \mid T_{l}^{*} h_{L}^{\prime}\right\rangle$ imply $\varepsilon \gamma=1+\left\|T_{a}\right\|^{2}$ and $\bar{\gamma}=\varepsilon$. Thus $|\varepsilon|$ $=|\gamma|=\sqrt{1+\left\|T_{a}\right\|^{2}}$. Let now $\varepsilon=\exp \{i 2 \theta\}|\varepsilon|$ with some $\theta \in\left[0,2 \pi\left[\right.\right.$, and define $h_{Q}$ 
$:=\exp \{i \theta\} h_{Q}^{\prime}$ and $h_{L}:=\exp \{-i \theta\} h_{L}^{\prime}$ and $v_{L}:=\exp \{-i \theta\}$.

As an immediate consequence of part (c) of the above Theorem we have the

Corollary 5.6. Assume $T \in \mathscr{T}(E)$, and let $L: E \rightarrow \mathbb{C}$ be a non-zero linear form. Let one of the following conditions be fulfilled:

(a) L is unbounded.

(b) $T_{a}$ (or equivalently $T_{l}$ ) is unbounded.

(c) $\operatorname{dim}\left(T_{a}(E)\right) \geq 2$.

(d) $\operatorname{dim}\left(T_{a}(E)\right)=1$ and $L$ is bounded with $L(f)=\langle h \mid f\rangle \forall f \in E$ for some non-zero $h \notin T_{a}(E)$.

Then it holds $\left.T_{a}^{*}\right|_{\operatorname{ker}(L)} \neq 0$, and we are in the situation of Theorem $5.5(b)$.

Thus the case (c) of Theorem 5.5 may happen for microscopic coherence (i.e., for bounded $L$ ), only. Especially for $\operatorname{dim}(E)=1$ we always have $\{0\}=\operatorname{ker}(L) \subseteq$ $\operatorname{ker}\left(T_{a}^{*}\right)$ for every non-zero linear form $L$. Detailed investigations of one-mode squeezing are found in the next section. Here let us only mention: In contrast to Theorem 5.5 (b) the case 5.5 (c) allows that the squeezing operation $\nu_{T}$ maps classical first order coherent states onto classical first order coherent states.

\section{§6. One-MHode Transformations}

Throughout the present Section we assume the case of one-mode squeezing as in Theorem $5.5(\mathrm{c})$ : The (bounded) symplectic $T=T_{l}+T_{a} \in \mathscr{T}(E)$ be defined in terms of the normalized vectors $h_{L}, h_{Q} \in E$ and the squeezing strength $\beta=\left\|T_{a}\right\| \geq 0$, such that the following characterizing relations are valid:

- $T_{a} f=\beta\left\langle f \mid h_{Q}\right\rangle h_{L}$, resp. $T_{a}^{*} f=\beta\left\langle f \mid h_{L}\right\rangle h_{Q}$, for all $f \in E$;

- $\bar{T}_{l}$ is a bijective isometry from $h_{Q}{ }^{\perp}$ onto $h_{L}{ }^{\perp}$;

- $T_{l} h_{Q}=\sqrt{1+\beta^{2}} h_{L}$, and $T^{*}{ }_{l} h_{L}=\sqrt{1+\beta^{2}} h_{Q}$.

However, since $\ell_{c l}^{L}=\oiint_{c l}^{v L}$ for each $0 \neq v \in \mathbb{C}$ by Corollary $5.4(\mathrm{a})$, we may choose the linear form $L: E \rightarrow \mathbb{C}$ here in contrast to Theorem 5.5 (c) simply as - $L(f)=\left\langle h_{L} \mid f\right\rangle$ for all $f \in E$.

Furthermore, let $Q: E \rightarrow \mathbb{C}$ be the linear form associated with $h_{Q}$, that is, $\circ Q(f)=\left\langle h_{Q} \mid f\right\rangle$ for all $f \in E$.

Theorem 6.1. Let all be as above, but $\beta \neq 0$. The subsequent assertions are valid:

(a) It holds $\nu_{T}\left(\oiint_{c L}^{L}\right) \cap \oiint_{c l} \subset \bigotimes_{c l}^{Q}$. For $\omega \in \bigotimes_{c l}^{L}$ the following conditions are equivalent:

(i) $\nu_{T}(\omega) \in \mathscr{S}_{c l}$, and thus $\nu_{T}(\omega) \in \mathscr{S}_{c l}^{Q}$;

(ii) $N_{\omega}^{T}$ : $\mathbb{C} \rightarrow \mathbb{C}$ is a positive-definite function on the additive group $\mathbb{C}$, where

$$
N_{\omega}^{T}(v):=\exp \left\{\frac{1}{2}\left[\beta^{2} \bar{v} v-\beta \sqrt{1+\beta^{2}} \operatorname{Re}\left(v^{2}\right)\right]\right\} \int_{b \mathbf{C}} \exp \{i \sqrt{2} \operatorname{Re}(z v)\} \mathrm{d} \mu_{\omega}^{L}(z) .
$$


(b) Let $\omega \in \mathscr{\&}_{c l}^{L}$ so that $\nu_{T}(\omega) \in \mathscr{\&}_{c l}^{Q}$, both being of class $\mathscr{C}^{2 m}$ for some $m \in \mathbb{N}$. Then

$$
c_{\nu_{T}(\omega)}^{Q}(k, l)=\left.(-i \sqrt{2})^{k+l} \frac{\partial^{k+l} M_{\omega}^{T}}{\partial v^{k} \partial \bar{v}^{-l}}\right|_{v=0}, 0 \leq k, l \leq m,
$$

where $M_{\omega}^{T}: \mathbb{C} \rightarrow \mathbb{C}$ is given by

$$
\begin{aligned}
& M_{\omega}^{T}(v):= \\
& \quad \exp \left\{-\frac{1}{2}\left[\beta^{2} \bar{v} v+\beta \sqrt{1+\beta^{2}} \operatorname{Re}\left(v^{2}\right)\right]\right\} \int_{\mathrm{C}} \exp \left\{i \sqrt{2} \operatorname{Re}\left(v \sqrt{1+\beta^{2}} z+\beta \bar{z}\right)\right\} \mathrm{d} \mu_{\omega}^{L}(z) .
\end{aligned}
$$

The moments $c_{\nu_{T}(\omega)}^{Q}(k, l)$ are thus obtained in terms of the moments $c_{\omega}^{L}(k, l)$, especially

$$
\begin{aligned}
& c_{\nu T(\omega)}^{Q}(1,0)=\sqrt{1+\beta^{2}} c_{\omega}^{L}(1,0)+\beta c_{\omega}^{L}(0,1), \\
& c_{\nu T(\omega)}^{Q}(1,1)=\beta^{2}+\beta \sqrt{1+\beta^{2}}\left(c_{\omega}^{L}(2,0)+c_{\omega}^{L}(0,2)\right)+\left(2 \beta^{2}+1\right) c_{\omega}^{L}(1,1), \\
& c_{\nu_{T}(\omega)}^{Q}(2,0)=\beta \sqrt{1+\beta^{2}}+\left(1+\beta^{2}\right) c_{\omega}^{L}(2,0)+\beta^{2} c_{\omega}^{L}(0,2)+2 \beta \sqrt{1+\beta^{2}} c_{\omega}^{L}(1,1) .
\end{aligned}
$$

If in addition $\omega$ is analytic, then $\omega \in \mathscr{\bigotimes}_{\lambda L}^{(1)} \cap \mathscr{S}_{c l}$ and $\nu_{T}(\omega) \in \mathscr{\bigotimes}_{x Q}^{(1)} \cap \mathscr{\ell}_{c l}$ with $|\lambda|^{2}=c_{\omega}^{L}(1,1)$ and $|\kappa|^{2}=c_{\nu_{T}(\omega)}^{Q}(1,1)$, by Corollary $5.4(c)$.

(c) For every $\lambda \in \mathbb{C}$ with $|\lambda|^{2} \geq \frac{1}{2}\left(\beta^{2}+\beta \sqrt{1+\beta^{2}}\right)$ it holds $\nu_{T}\left(\mathscr{\bigotimes}_{\lambda L}^{(1)} \cap \mathscr{\&}_{c l}\right) \cap \mathscr{\&}_{c l} \neq \emptyset$, that is, there exist some $\omega \in \mathscr{\bigotimes}_{\lambda L}^{(1)} \cap \mathscr{S}_{c l}\left(\right.$ implying $\left.c_{\omega}^{L}(1,1)=|\lambda|^{2}\right)$ so that $\nu_{T}(\omega) \in$

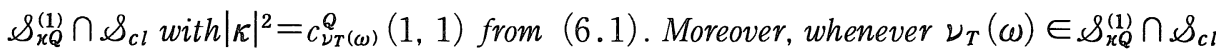
for some (analytic) $\omega \in \mathscr{X}_{\text {cl }}^{L}$ and some $\kappa \in \mathbb{C}$, then $|\kappa|^{2} \geq \frac{1}{2}\left(\beta^{2}+\beta \sqrt{1+\beta^{2}}\right)$.

(d) For every $\lambda \in \mathbb{C}$ with $|\lambda|^{2}<\frac{1}{2}\left(\beta^{2}+\beta \sqrt{1+\beta^{2}}\right)$ it holds $\nu_{T}\left(\oiint_{\lambda L}^{(1)} \cap \oiint_{c l}\right) \cap \oiint_{c l}=\emptyset$.

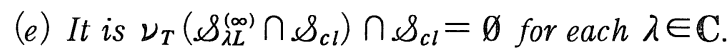

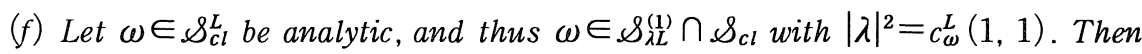
$\nu_{T}(\omega) \in \oiint_{x Q}^{(1)}$ with $(c f$. equation $(6.1))$

$$
|\kappa|^{2}=\beta^{2}+\beta \sqrt{1+\beta^{2}}\left(c_{\omega}^{L}(2,0)+c_{\omega}^{L}(0,2)\right)+\left(2 \beta^{2}+1\right) c_{\omega}^{L}(1,1) .
$$

Observe, that by the parts $(c)(d)$, and $(e)$ the transformed $\nu_{T}(\omega)$ in many cases is a non-classical first order coherent state.

Proof. (a) and (b): Let $\omega \in \mathscr{\bigotimes}_{c l}^{L}$ such that $\nu_{T}(\omega) \in \mathscr{\bigotimes}_{c l}$. Then by equations (2.12) and (5.1) we obtain the positive-definite function

$$
P_{\nu T(\omega)}(f)=\exp \left\{\frac{1}{4}\left(\|f\|^{2}-\|T f\|^{2}\right)\right\} \int_{b \mathbf{C}} \exp \{i \sqrt{2} \operatorname{Re}(z L(T f))\} \mathrm{d} \mu_{\omega}^{L}(z)=M_{\omega}^{T}(Q(f)) .
$$

Consequently $\nu_{T}(\omega) \in \mathscr{S}_{c l}^{Q}$ and $M_{\omega}^{T}(v)=\int_{b \mathbf{C}} \exp \{i \sqrt{2} \operatorname{Re}(v z)\} \mathrm{d} \mu_{\nu_{T}(\omega)}^{Q}(z)$. From 
Subsection 2.3 we know that $\omega$ is regular, of class $\mathscr{C}^{m}$, analytic, entire-analytic, if and only if $\nu_{T}(\omega)$ is so. Hence (b) follows from Theorem 5.3 (b) and Corollary 5.4 .

(c), (d), and (a) (i) $\Leftrightarrow(\mathrm{a})(\mathrm{ii})$ : Applying equation (2.12) to $T^{-1}=T_{l}^{*}-T_{a}^{*}$ yields $N_{F}^{T}(f) P_{\omega}(f)=N_{\omega}^{T}(v)$ with $v:=L(f)$. Thus (a) (ii) is equivalent to $N_{F}^{T} P_{\omega} \in$ $\mathbb{P}(E)$, which by Lemma 3.4 is equivalent to (a) (i). Let $x:=\operatorname{Re}(v)$ and $y:=$ $\operatorname{Im}(v)$. If $\nu_{T}(\omega) \in \mathscr{S}_{c l}^{Q}$ is of class $\mathscr{C}^{2}$, then $-\frac{\partial^{2} N^{T}}{\partial y^{2}}$ is also positive-definite, which especially implies

$$
0 \leq-\left.\frac{\partial^{2} N_{\omega}^{T}}{\partial y^{2}}\right|_{v=0}=2 \int_{\mathbb{C}}(\operatorname{Im}(z))^{2} \mathrm{~d} \mu_{\omega}^{L}(z)-\left(\beta^{2}+\beta \sqrt{1+\beta^{2}}\right),
$$

which in turn implies $c_{\omega}^{L}(1,1) \geq \int_{\mathbf{C}}(\operatorname{Im}(z))^{2} \mathrm{~d} \mu_{\omega}^{L}(z) \geq \frac{1}{2}\left(\beta^{2}+\beta \sqrt{1+\beta^{2}}\right)$ and hence $(d)$. Now for $a \geq 0$ there obviously exists a $\mu_{\omega_{a}}^{L} \in \mathbb{M}_{+}^{1}(\mathbb{C})$ concentrated on $\{z$ $\in \mathbb{C} \mid \operatorname{Re}(z)=0\}$ so that for each $\operatorname{Im}(v)=y \in \mathbb{R}$

$$
\int_{C} \exp \{-i \sqrt{2} \operatorname{Im}(z) y\} \mathrm{d} \mu_{\omega_{a}}^{L}(z)=\exp \left\{-\frac{a}{2}\left(\beta^{2}+\beta \sqrt{1+\beta^{2}}\right) y^{2}\right\},
$$

which gives the quasifree state $\omega_{a} \in \mathscr{S}_{c l}^{L}$ of Example 6.2 below. Differentiating (6.3) with respect to $y \in \mathbb{R}$ yields $(6.4)$, especially $c_{\omega_{a}}^{L}(1,1)=\frac{a}{2}\left(\beta^{2}+\beta\right.$ $\left.\sqrt{1+\beta^{2}}\right) \cdot N_{\omega a}^{T}$ is positive-definite, if and only if $a \geq 1$. Hence $\nu_{T}\left(\omega_{a}\right) \in \mathscr{Q}_{c l}^{Q}$ for all $a \geq 1$, which with Theorem 5.3 and Corollary 5.4 gives (c). Now apply the results so far to $T^{-1}$ to obtain, that in any case $|\kappa|^{2} \geq \frac{1}{2}\left(\beta^{2}+\beta \sqrt{1+\beta^{2}}\right)$.

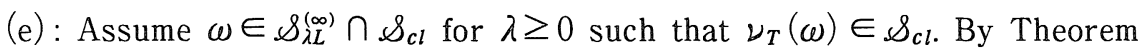
5.3 (d) and Corollary 5.4 (b) $\mu_{\omega}^{L}$ is supported by $\{z \in \mathbb{C}|| z \mid=\lambda\}$. By assumption it follows from (a) that $N_{\omega}^{T}$ is positive-definite, and so is its restriction to $i \mathbb{R}=\{z \in$ $\mathbb{C} \mid \operatorname{Re}(z)=0\} \subset \mathbb{C}$. But

$$
\int_{\mathbb{C}} \exp \{i \sqrt{2} \operatorname{Re}(z i p)\} \mathrm{d} \mu_{\omega}^{L}(z)=\int_{\mathbf{C}} \exp \{-i \sqrt{2} \operatorname{Im}(z) p\} \mathrm{d} \mu_{\omega}^{L}(z)=: \hat{\rho}(p), p \in \mathbb{R},
$$

is the Fourier transform of the measure $\rho \in \mathbb{M}_{+}^{1}([-\lambda, \lambda])$, which is the projection of $\mu_{\omega}^{L}$ on the complex axis $i \mathbb{R} \cong \mathbb{R}$. Consequently, $p \in \mathbb{R} \mapsto N_{\omega}^{T}(i p)=$ $\exp \left\{\frac{1}{2}\left(\beta^{2}+\beta \sqrt{1+\beta^{2}}\right) p^{2}\right\} \hat{\rho}(p)$ is positive-definite, and thus given by the Fourier transform $\widehat{\sigma}$ of a measure $\sigma \in \mathbb{M}_{+}^{1}(\mathbb{R})$ (Bochner's theorem). $\exp \left\{-\frac{1}{2}\left(\beta^{2}+\right.\right.$ $\left.\left.\beta \sqrt{1+\beta^{2}}\right) p^{2}\right\}$ is the Fourier transform of a Gaussian function $\phi$ on $\mathbb{R}, \phi(k)>0$ 
$\forall k \in \mathbb{R}$ (Lebesgue measure $\ell$ ). Hence $\hat{\rho}=\widehat{\phi} \widehat{\sigma}=\widehat{\phi * \sigma}$, resp. $\rho=\phi * \sigma$ with the convolution $*$, which yields $\rho(B)=\int_{B} \int_{\mathbf{R}} \psi(k-q) \mathrm{d} \sigma(q) \mathrm{d} \boldsymbol{\ell}(k)>0$ for every Borel set $B \subseteq \mathbf{R}$ with $\int_{B} \mathrm{~d} \boldsymbol{\ell}(k) \neq 0$. But $\rho$ is supported by $[-\lambda, \lambda]$, a contradiction.

(f): Inserting our specific form for $T_{a}, T_{l}$, and $L$ into (5.5) gives $\left\langle\nu_{T}(\omega)\right.$; $\left.a^{*}(f) a(f)\right\rangle=|\kappa|^{2}|Q(f)|^{2} \forall f \in E$. The polarisation identity for the positive hermitian form $(g, f) \in E \times E \mapsto\left\langle\nu_{T}(\omega) ; a^{*}(f) a(g)\right\rangle$ yields $\left\langle\nu_{T}(\omega) ; a^{*}(f) a(g)\right\rangle=$ $\overline{\kappa Q(g)} \kappa Q(f) \forall f, g \in E$, that is, $\nu_{T}(\omega) \in \mathscr{\ell}_{x Q}^{(1)}$.

From the above proof we obtain the following example.

Example 6.2. Let all be as above. For each $a \geq 0$ there is a unique state $\omega_{a}$ $\in \mathscr{\&}_{c l}^{L}$ with

$$
P_{\omega a}(f)=\exp \left\{-\frac{a}{2}\left(\beta^{2}+\beta \sqrt{1+\beta^{2}}\right)[\operatorname{Im}(L(f))]^{2}\right\} \forall f \in E .
$$

$\omega_{a}$ is quasifree, $\mu_{\omega_{a}}^{L} \in M_{+}^{1}(\mathbf{C})$ is concentrated on $\{z \in \mathbf{C} \mid \operatorname{Re}(z)=0\}$, with the new moments

$$
c_{\omega a}^{L}(1,0)=0, c_{\omega_{a}}^{L}(1,1)=\frac{a}{2}\left(\beta^{2}+\beta \sqrt{1+\beta^{2}}\right)=-c_{\omega_{a}}^{L}(2,0)
$$

We have $\nu_{T}\left(\omega_{a}\right) \in \oiint_{c l}$ (which is equivalent to $\left.\nu_{T}\left(\omega_{a}\right) \in \mathscr{\bigotimes}_{c l}^{Q}\right)$, if and only if $a \geq 1$, in which case he moments are given by

$$
\begin{aligned}
& c_{\nu T\left(\omega_{a}\right)}^{Q}(1,0)=0, c_{\nu T\left(\omega_{a}\right)}^{Q}(1,1)=\beta^{2}+\frac{a}{2}\left(\beta \sqrt{1+\beta^{2}}-\beta^{2}\right), \\
& c_{\nu T\left(\omega_{a}\right)}^{Q}(2,0)=\beta \sqrt{1+\beta^{2}}-\frac{a}{2}\left(\beta \sqrt{1+\beta^{2}}-\beta^{2}\right) .
\end{aligned}
$$

As an immediate consequence of the parts (c), (d), and (f) of Theorem 6.1 we get the

Corollary 6.3. Let us here fix a $\lambda>0$ and consider the classical, first order coherent states $\oiint_{\lambda L}^{(1)} \cap \oiint_{c l}$. By Theorem $6.1(c)$ and $6.1(d)$ there exists the critical squeezing strength $\beta_{c} \equiv \beta_{c}(\lambda)>0$, which is given by

$$
\beta_{c}^{2}+\beta_{c} \sqrt{1+\beta_{c}^{2}}:=2|\lambda|^{2} .
$$

If the actual squeezing strength $\beta$ is below the critical valve, $0 \leq \beta \leq \beta_{c}$, then some states $\omega \in \mathscr{S}_{\lambda L}^{(1)} \cap \mathscr{S}_{c l}$ remain classical under the one-mode squeezing transformation $T \equiv T(\beta) \in \mathscr{T}(E)$, that is, $\nu_{T}(\omega) \in \oiint_{c l}$. For strong squeezing, i.e., for $\beta$ above the critical value, $\beta>\beta_{c}$, however, each $\omega \in \mathscr{\bigotimes}_{\lambda L}^{(1)} \cap \mathscr{\bigotimes}_{c l}$ is rendered non-classical by the squeezing $T=T(\beta)$, that is, $\nu_{T}(\omega) \notin \mathscr{\&}_{c l}$.

But it holds for arbitrary squeezing strength $\beta \geq 0$, that for every $\omega \in \mathscr{\bigotimes}_{\lambda L}^{(1)} \cap \mathscr{\bigotimes}_{c l}$ the transformed (squeezed) state $\nu_{T}(\omega)$ is first order coherent. The associated 
factorizing linear form is given by $\kappa Q: E \rightarrow \mathbb{C}$ for a value $\kappa \equiv \kappa(\omega, \beta) \in \mathbb{C}$ depending on $\beta$ and on the initial state $\omega \in \mathscr{\bigotimes}_{\lambda L}^{(1)} \cap \mathscr{\bigotimes}_{c l}$ according to equation (6.2).

\section{Acknow Hedgement}

This work has been supported by the Deutsche Forschungsgemeinschaft.

\section{Reiferences}

[1] Araki, H. and Shiraishi, M.. On Quasifree States of the Canonical Commutation Relations I, Publ. RIMS, Kyoto Univ., 7 (1971/72), 105-120.

[2] Araki. H.. On Quasifree States of the Canonical Commutation Relations II. Prubl. RIMS, Kyoto Univ.. 7(1971/72), 121-152.

[3] Araki, H. and Woods, E. J., Representations of the Canonical Commutation Relations Describing a Nonrelativistic Infinite Free Bose Gas. J. Math. Phys., 4 (1963), 637-662.

[4] Bratteli. O. and Robinson, D. W., Operator Algebras and Quantum Statisiical Mechanics II. Springer Verlag, Berlin, New York, 1981.

[5] Brendle, R., Einstein Condensation in a Macroscopic Field. Z. Naturforsch., 40a (1985). 1189-1198.

[6] Cannon, J. T., Infinite Volume Limits of the Canonical Free Bose Gas States on the Weyl Algebra. Comm. Math. Phys., 29 (1973), 89-104.

[7] Cohen-Tannoudji, C., Dupont-Roc, J. and Grynberg, G.. Photons \& Atoms, Introduction to QED. Wiley \& Sons. New York, 1989.

[8] Evans, D. E. and Lewis, J. T., Dilations of Irreversible Evolutions in Algebraic Quantum Theory, Comm. Dublin Ins. Adv. Stud. Ser. A, Theoreet. Phys. No. 24. Dublin, 1977.

[9] Glauber, R. J.. The Quantum Theory of Optical Coherence, Phys. Rev., 130 (1963). 2529-2539. 2766-2788 . Coherent and Incoherent States of the Radiation Field. Phys. Rev.. 131 (1963). , in Quantum Optics and Electronics, Les Houches 1964, edited by C. de Witt, A. Blandin and C. Cohen-Tannoudji, Gordon and Breach, New York, 1965.

[10] Haken. H., Light 1. North-Holland Publishing Company, Amsterdam, 1981.

[11] Hertle, J., Macroscopically Inhomogeneous Bose-Einstein Condensation, p. 339-344, in LargeScale Molecular Systems: Quantum and Stochastic Aspects. Beyond the Simple Molecular Picture. NATO ASI, edited by W. Gans, A. Blumen, and A. Amann, Plenum Press Series B. Physics, 258. 1991.

[12] Hertle, J. and Honegger, R., Limiting Gibbs States and Dynamics for Thermal Photons, J. Math. Phys., 33 (1992), 143-151.

[13] Hewitt, E. and Ross, K. A., Abstract Harmonic Analysis I, II, Springer Verlag, New York, 1963. 1970.

[14] Honegger, R., Decomposition of Positive Sesquilinear Forms and the Central Decomposition of Gauge-Invariant Quasi-Free States on the Weyl Algebra, Z. Naturforsch., 45a (1990), 17-28.

[15] _ Globale Quantentheorie der Strahlung. Doctoral Thesis, Institut fur Theoretische Physik, Universität Tübingen, Tübingen. 1991.

[16] . On Heisenberg's Uncertainty Principle and the CCR, Z. Naturforsch., 48a (1993). 447-451.

[17] . The General Form of the Microscopic Coherent Boson States, Physica A, 198 (1993), 
179-209.

[18] Honegger, R., The Extremal Microscopic Coherent Boson States, Lett. Math. Phys., 28 (1993), $155-164$.

[19] Honegger, R. and Rapp. A., General Glauber Coherent States on the Weyl Algebra and their Phase Integrals, Physica A, 167 (1990), 945-961.

[20] Honegger, R. and Rieckers, A., The General Form of Non-Fock Coherent Boson States, Publ. RIMS, Kyoto Univ.. 26 (1990), 397-417.

[21] - First Order Coherent Boson States, Helv. Phys. Acta, 65 (1992), 965-984.

[22] On Higher Order Coherent States on the Weyl Algebra, Lett. Math. Phys., 24 (1992). 221-225.

[23] Construction of Classical and Non-Classical Coherent Photon States. prepint, Tubingen. (1998).

[24] Squeezing Bogoliubov Transformations on the Infinite Mode CCR-Algebra, J. Math Phys., 37 (1996) , 4292-4309.

[25] _. Squeezing Operations in Fock Space and Beyond, Physica A, 24 (1997), 423-438.

[26] _. Squeezed Variances of Smeared Boson Fields, Helv. Phys. Acta. 70 (1997), 507-541.

[27] Unitary Implementations of One-Parameter Squeezing Groups, to appear in J. Math. Phys., (1998).

[28] Lewis. J. T. and Pulè, J. V.. The Equilibrium States of the Free Boson Gas, Commun. Math. Phys., 36 (1974), 1-18.

[29] Loudon, R., The Quantum Theory of Light, Clarendon Press, Oxford, 1979.

[30] Manuceau, J. and Verbeure, A., Quasi-Free States of the C.C.R.-Algebra and Bogoliubov Transformations, Commun. Math. Phys., \$(1968), 293-302.

[31] Meysire, P. and Sargent, M. III, Elements of Quantum Optics, Springer Verlag, Berlin, New York, 1990.

[32] Nussenzveig, H. M., Introduction io Quantum Optics, Gordon and Breach, London, New York, 1973.

[33] Rieckers, A., Classical States of Quantized Boson Fields, in Proceedings of the V International Conference on Selected Topics in Quantum Field Theory and Mathematical Physics. Liblice, Czechoslovakia. June 1989, edited by J. Niederle and J. Fischer. World Scientific, Singapore, New Jersey, London, Hong Kong, 1990.

[34] Robinson, D. W.. The Ground State of the Bose Gas, Comm. Math. Phys., 1 (1965). 159-174.

[35] Shale. D. and Stinespring, W. F.. States on the Clifford Algebra. Ann. Math., 80 (1964), 365-381.

[36] Testard, D., Les Etat Quasi-Libre du Champ des Bosons considerée comme Eiats d'Equilibre à l'Aide de la Condition de Kubo, Martin et Schwinger, Thèse de Doctorat d'Etat. Centre de Physique Théorique, Faculté des Sciences de Marseille, No. d’Ordre: A.O. 3680, Marseille, 1970.

[37] Titulaer, U. M. and Glauber, R. J., Density Operators for Coherent Fields, Phys. Rev.. 145 (1966). 1041-1050.

[38] Yuen, H. P., Generalized Coherent States and the Statistics of Two-Photon-Lasers, Phys. Lett. A, 51 (1975), 1-2.

, Two-Photon Coherent States of the Radiation Field, Phys. Rev. A, 13 (1976), 2226-2243.

Yuen. H. P. and Shapiro, J. H., Optical Communication with Two-Photon Coherent States, Part I in IEEE Trans. Inform. Theory, IT-24 (1978), 657-668, Part II in Optics Letters, 4 (1979), 
334-349, and Part III in IEEE Trans. Inform. Theory, IT-26 (1980), 78-93.

Walls, D. F., Squeezed States of Light, Nature, 306 (1983), 141-146.

Loudon, R. and Knight, P. L., Squeezed Light, J. Mod. Opt., 34 (1987), 709-759.

Huang, J. and Kumar, P., Photon-Counting Statistics of Multimode Squeezed Light, Phys. Rev. A, 40 (1989), 1670-1673.

Ma, X. and Rhodes, W., Multimode Squeeze Operators and Squeezed States, Phys. Rev, A, 41 (1990), 4625-4631.

Ekert, A. K. and Knight. P. L., Relationship between Semiclassical and Quantum-Mechanical Input-Output Theories of Optical Response, Phys. Rev. A, 43 (1991), 3934-3938. 